IZA DP No. 7271

Authority and Incentives in Organizations

Matthias Kräkel

March 2013

Forschungsinstitut zur Zukunft der Arbeit Institute for the Study of Labor 


\title{
Authority and Incentives in Organizations
}

\author{
Matthias Kräkel \\ University of Bonn \\ and IZA
}

\author{
Discussion Paper No. 7271 \\ March 2013
}

\author{
IZA \\ P.O. Box 7240 \\ 53072 Bonn \\ Germany \\ Phone: $+49-228-3894-0$ \\ Fax: +49-228-3894-180 \\ E-mail: iza@iza.org
}

Any opinions expressed here are those of the author(s) and not those of IZA. Research published in this series may include views on policy, but the institute itself takes no institutional policy positions. The IZA research network is committed to the IZA Guiding Principles of Research Integrity.

The Institute for the Study of Labor (IZA) in Bonn is a local and virtual international research center and a place of communication between science, politics and business. IZA is an independent nonprofit organization supported by Deutsche Post Foundation. The center is associated with the University of Bonn and offers a stimulating research environment through its international network, workshops and conferences, data service, project support, research visits and doctoral program. IZA engages in (i) original and internationally competitive research in all fields of labor economics, (ii) development of policy concepts, and (iii) dissemination of research results and concepts to the interested public.

IZA Discussion Papers often represent preliminary work and are circulated to encourage discussion. Citation of such a paper should account for its provisional character. A revised version may be available directly from the author. 


\section{ABSTRACT}

\section{Authority and Incentives in Organizations*}

The paper analyzes how the choice of organizational structure leads to the best compromise between controlling behavior based on authority rights and minimizing costs for implementing high efforts. Concentrated delegation and hierarchical delegation turn out to be never an optimal compromise. If the CEO is more efficient than the division heads (i.e., the CEO's costs from exerting high effort are smaller than those of the division heads), the owner will prefer full delegation to the divisions to replace high incentive pay to the division heads by incentives based on private benefits of control. In that situation, decentralization is the optimal form of full delegation given that selfish behavior is more important than cooperation, but cross-authority delegation is optimal for cooperation being crucial. If, however, the division heads are clearly more efficient than the CEO, the owner will choose centralization given that cooperation is the dominating issue, but partial delegation if selfish behavior is crucial.

JEL Classification: D21, D23, D86, L22

Keywords: authority, centralization, contracts, decentralization, moral hazard

Corresponding author:

Matthias Kräkel

Department of Economics

University of Bonn

Adenauerallee 24-42

53113 Bonn

Germany

E-mail:m.kraekel@uni-bonn.de

\footnotetext{
* Financial support by the Deutsche Forschungsgemeinschaft (DFG), grant SFB/TR 15, is gratefully acknowledged.
} 


\section{Introduction}

In many environments, hierarchies have evolved as optimal organizational form to deal with complex tasks (e.g., Chandler 1977, Williamson 1981). Concerning economic activities, we can observe large corporations that are controlled by a central decision maker - the Chief Executive Officer (CEO) - at the top. This central decision maker determines the business strategy of the corporation and exerts effort that influences the performance of all organizational units at lower hierarchy levels. Complexity of tasks arises from the division of labor within the corporation and from complementarities between the organizational units (e.g., Milgrom and Roberts 1995a, 1995b).

Given such raw form of a hierarchy, the fundamental question then is how should authority rights be allocated between the CEO and the organizational units to create the best organizational structure? In this paper, I will give an answer from incentive perspective. I consider a stylized hierarchy model with four players - the owner of the corporation, who chooses optimal incentive contracts for the three other players and decides on the allocation of authority rights, two division heads, who choose efforts to increase the success probability of their organizational units or divisions, and the CEO or top manager whose effort choice influences the performance of both divisions.

Besides the top manager's effort choice there is a second externality in the model, which exists between the two divisions. If one division is successful and behaves cooperatively, this success will also contribute to the returns of the other division. For example, suppose that one division produces certain goods and the other division sells these goods. On the one hand, the production department's decision which technology to use (e.g., applying a standard or an innovative production technique) influences the timing, quality and degree of diversity of produced output, which influences the success of the sales division. On the other hand, the sales division's choice of distributive channels influences both own sales and the internal demand for output from the production department. Similar externalities can arise between two divisions if one division produces an intermediate good or service that is used by the 
other division for producing a consumer good and selling it to customers.

This paper combines the organizational-design setting of Choe and Ishiguro (2012) with the moral-hazard limited-liability approach to analyze two kinds of incentives. First, following Choe and Ishiguro (2012), I assume that players receive private benefits of control from having decision authority over a division, which incentivizes the players as their private benefits increase in the performance of the division. Second, inspired by Che and Yoo (2001), Laffont and Martimort (2002), Hermalin (2005), and Schmitz (2005a, 2013), among others, I use a binary effort moral-hazard model to solve for the optimal incentive contracts for the top manager and the two division heads. In particular, I assume that the performance of each division is observable and contractible so that success and failure of the two divisions can be used to employ an explicit incentive scheme.

As in the paper by Choe and Ishiguro, there are six possible allocations of authority rights leading to six different organizational structures. (1) If the top manager receives the decision rights for both divisions, we will obtain a centralized organization. (2) Alternatively, all decision rights can be given to one of the division heads, leading to concentrated delegation. (3) A three-tier hierarchy, labeled hierarchical delegation, arises if the top manager has decision authority over one division whose head possesses the decision rights over the other division. (4) We can speak of partial delegation, if one division head has authority over his own division but the top manager decides on the other division. (5) Decentralization will exist, if each division head decides on his own division. (6) If each division head has decision rights over the other division, respectively, the organizational structure can be called cross-authority delegation. In this paper, I analyze how the interplay of incentives from private benefits of control and from the optimal incentive contract determines the owner's choice of the profit maximizing organizational structure.

Allocating decision authority to the top manager and/or the division heads provides them with incentives, which do not directly lead to labor costs for the owner. Consequently, the owner uses these incentives to re- 
place incentives based on pay for performance, which would imply positive labor costs. The allocation of authority also influences the players' decisions towards more selfish or more cooperative behavior, respectively. For example, under decentralization each division head solely cares for his own division, which fosters selfish behavior and works against cooperation. As a main finding of the paper, the owner will choose the organizational structure that leads to the best compromise between controlling behavior based on authority rights and saving labor costs.

I will show that, if pay for performance is still necessary to implement high efforts, under any organizational structure the owner will choose incentive contracts for the manager and the division heads that are based on overall firm performance. Such contracts are optimal to exploit the positive externalities from both the manager's effort choice and the interplay of the two divisions. If the division heads are less efficient than the manager (i.e., the division heads' costs from exerting high effort are larger than those of the manager), explicit pay for performance for motivating the division heads would be quite high. In this situation, the owner prefers either decentralization or cross-authority delegation to replace the division heads' monetary incentives by incentives based on private benefits of control. Decentralization (cross-authority delegation) will be optimal if selfish (cooperative) behavior of the two divisions is more important for maximizing firm profits. If, however, the manager is clearly less efficient than the division heads (i.e., the manager's costs from exerting high effort are considerably larger than those of the division heads), either centralization or partial delegation will be the optimal organizational structure. In that situation, centralization (partial delegation) has a comparative advantage if cooperation (selfish behavior) is crucial for high performance of the whole firm. Concentrated delegation and hierarchical delegation are never optimal. The following table roughly summarizes the main findings on the optimal organizational structure: 


\begin{tabular}{|c|c|c|}
\cline { 2 - 3 } \multicolumn{1}{c|}{} & $\begin{array}{l}\text { selfish behavior } \\
\text { important }\end{array}$ & $\begin{array}{c}\text { cooperation } \\
\text { important }\end{array}$ \\
\hline $\begin{array}{l}\text { division heads } \\
\text { less efficient } \\
\text { than manager }\end{array}$ & decentralization & $\begin{array}{l}\text { cross-authority } \\
\text { delegation }\end{array}$ \\
\hline $\begin{array}{l}\text { manager clearly } \\
\text { less efficient than } \\
\text { division heads }\end{array}$ & $\begin{array}{l}\text { partial } \\
\text { delegation }\end{array}$ & centralization \\
\hline
\end{tabular}

The paper is related to two strands of the literature. First, there are parallels to the literature on organizational design and the allocation of authority rights in organizations. Dessein (forthcoming) gives an overview on this literature and discusses new directions. The seminal paper by Aghion and Tirole (1997) introduces delegation of authority to subordinate managers as an incentive device. Aghion et al. (2002) investigate in a partial-contracting setting how delegation of authority is optimally used for inducing cooperative behavior. Dessein (2002) addresses the problem of communication in hierarchies and shows under which conditions delegation to an intermediate party can be optimal. Alonso et al. (2008) and Rantakari (2008) also consider communication in organizations and focus on the fundamental trade-off between adaptation and coordination. On the one hand, divisions have to be adapted to local conditions in order to be effective. On the other hand, high firm performance requires close coordination of the divisions' activities, which can lead away from best adaptation. Alonso et al. (2008) and Rantakari (2008) analyze the conditions for decentralization or centralization being a better solution to the given trade-off. Under decentralization, division heads directly communicate with each other and then decide for their divisions, whereas under centralization the division heads communicate with the CEO who decides for the two divisions thereafter. Thiele (2013) also compares decentralization to centralization as optimal organizational structures. However, he uses a setting in which the optimal structure has to 
solve the trade-off between more accurate information from subjective performance evaluation and possible collusion between the agents at lower tiers of the hierarchy. Here, centralization refers to a situation in which the principal evaluates the agents, whereas decentralization leads to the delegation of the evaluation task to a supervisor.

As indicated above, my paper is most closely related to Choe and Ishiguro (2012). They address the same six organizational structures and investigate which organization is optimal from the owner's point of view. However, in the model by Choe and Ishiguro, the manager's and division heads' incentives are exclusively exogenous. On the one hand, players have incentives based on private benefits of control from received decision authority, as in my paper. On the other hand, the manager is motivated by intrinsic concerns for firm success, whereas the division heads have intrinsic concerns for their respective divisions. In my paper, I show that replacing intrinsic motivation by optimal incentive contracts yields a new effect - namely, the allocation of authority rights as a means of substituting pay for performance by incentives from having authority. This new effect leads to completely different results compared to Choe and Ishiguro (2012). In particular, hierarchical delegation can be optimal in the setting of Choe and Ishiguro (2012) but is never optimal in my model. Moreover, in the model by Choe and Ishiguro, centralization (decentralization) will be optimal if the top manager is more efficient (less efficient) than the two division heads. These findings are just reversed in my setting due to the substitution effect explained before. Since both alternatives - intrinsic motivation and optimal endogenous incentives based on division performance - seem realistic, my analysis complements the one by Choe and Ishiguro by pointing to an important new effect under optimal contracts.

The second strand of related literature analyzes optimal contracts under moral hazard and limited liability. As emphasized by Laffont and Martimort (2002, Sections 4.3 and 4.4) there exist two standard frictions in contracting that inhibit the implementation of first-best incentives: if agents are either 
risk averse or protected by limited liability the implementation of first-best efforts will be too costly for the principal so that he prefers to implement lower effort under the optimal contract. Note that either friction leads to costs for the principal. In case of risk aversion and unlimited liability, the principal has to fully compensate the agent for the risk premium. In case of limited liability, the agent earns a positive rent which typically increases in the level of implemented effort. In this paper, I apply the second friction, which has been introduced by the seminal paper of Sappington (1983) and later used by many others to address incentive problems under moral hazard (e.g., Innes 1990, Demougin and Fluet 1998, Schmitz 2005a, 2005b, Ohlendorf and Schmitz 2012, Kräkel and Schöttner 2012). Within this class of models, my paper is closest to Schmitz (2013), who also uses a binary effort approach and looks for optimal incentives to implement the higher effort level. The approach by Schmitz is very useful since it leads to a clear characterization of the optimal contract without ad hoc restriction of the class of feasible contracts. Contrary to my paper, Schmitz (2013) does not consider the allocation of authority within organizations and the corresponding optimal contract. He analyzes two sequential production stages which are conflicting and for which the principal can hire either one agent or two agents. Under the optimal contract, the principal employs one agent for both tasks because the expected second-period rent increases in the agent's first-period success, which additionally motivates the agent in period one.

The paper is organized as follows. The next section introduces the model. In Section 3, I derive the optimal contract for any given organizational structure. In Section 4, the optimal wages and corresponding expected profits for the six possible organizational structures are computed. Section 5 compares the expected profits and presents the optimal organizational structure for all feasible parameter constellations. Section 6 concludes. 


\section{The Model}

In this paper, I combine the organizational design set-up of Choe and Ishiguro (2012) with the binary-effort moral-hazard approach of Schmitz (2013). I consider a firm that consists of four risk neutral parties - an owner $O$, a manager $M$, and two division heads $A$ and $B$. Owner $O$ decides on the organizational structure of the firm and chooses incentive contracts for the three other parties. Manager $M$ exerts effort $e_{M} \in\{0,1\}$ which leads to effort costs $k \cdot e_{M}$ with $k>0$. Division head $i(i=A, B)$ also chooses effort, denoted by $e_{i} \in\{0,1\}$ leading to $\operatorname{costs} c \cdot e_{i}$ with $c>0$. The three effort choices influence the performance of the two divisions and, hence, overall firm performance.

Division $i(i=A, B)$ is successful with probability $P_{i} \equiv P_{i}\left(e_{i}+e_{M}\right) \in$ $(0,1)$ and fails with probability $1-P_{i}\left(e_{i}+e_{M}\right)$. Hence, manager $M$ 's effort choice is a positive externality for both divisions. For example, if $M$ spends effort to improve overall firm reputation this will help both divisions in selling their products. To compute explicit solutions, I assume that each positive effort level $e_{i}=1$ or $e_{M}=1$ adds the probability mass $\rho>0$ to the success probability of division $i$ whereas zero effort adds zero probability to $P_{i}$ :

$$
P_{i}\left(e_{i}, e_{M}\right)=\left\{\begin{array}{ccc}
2 \rho & \text { if } & e_{i}+e_{M}=2 \\
\rho & \text { if } & e_{i}+e_{M}=1 \\
0 & \text { if } & e_{i}+e_{M}=0
\end{array}\right.
$$

with $2 \rho<1$. We have a moral hazard problem since the owner can observe the success of each division, which is also contractible, but does not observe the effort choices of the three other parties.

As Choe and Ishiguro (2012), I assume that the divisions are interconnected so that the success of one division also contributes to the returns of the other division. In particular, if division $A$ succeeds this will yield returns $h(a)$ for division $A$ and $q(a)$ for division $B$. Similarly, if division $B$ is successful, this outcome will increase the returns of division $B$ by $h(b)$ and the returns of division $A$ by $q(b)$. If a division fails, this will contribute zero 
returns to either division. As indicated by the notation, the specific returns depend on the endogenous decisions $(a, b)$ with $a \in\{\hat{S}, \hat{C}\}$ and $b \in\{\hat{S}, \hat{C}\}$. As will become clear from the following, " $\hat{S}$ " stands for selfish behavior and " $\hat{C}$ " for cooperative behavior. In addition to the specific returns introduced before, $h(\cdot)$ and $q(\cdot)$, each successful division yields basic returns $R>0$ that directly accrue to owner $O$.

The decision rights on $a$ and $b$ are allocated by the owner $O$ to the three other parties. For example, division head $A$ may obtain authority on $a$ and division head $B$ on $b$ so that we have decentralization as organizational structure, or all decision rights may be allocated to manager $M$ leading to a centralized organization. Altogether, there are $3^{2}=9$ alternative allocations of decision rights that can be chosen by the owner. Let the allocation of decision authority be denoted by $D:=\left\{\chi_{M j}, \chi_{A j}, \chi_{B j}\right\}_{j=A, B}$. The indicator variable $\chi_{i A}\left(\chi_{i B}\right)$ takes the value 1 if player $i$ has decision authority over division $A$ (division $B$ ) and, hence, chooses $a(b)$. However, $\chi_{i A}\left(\chi_{i B}\right)$ takes the value 0 if player $i$ is not allowed to choose $a(b)$.

To simplify matters, I follow Choe and Ishiguro (2012, p. 493) by assuming that $q(\hat{C}):=q>q(\hat{S})=0$ and $h(\hat{S}):=h>h(\hat{C})=0$. Hence, if a division is successful, selfish behavior by the authorized decision maker will add positive returns $h$ to this division, but zero returns to the other division. However, cooperative behavior increases the returns of the other division by $q$ but adds zero returns to the division for which the decision maker is responsible. Altogether, given efforts $\mathbf{e}=\left(e_{M}, e_{A}, e_{B}\right)$, the expected specific returns of division $A$ sum up to

$$
E\left[\pi_{A} \mid \mathbf{e}\right]=P_{A} \cdot h(a)+P_{B} \cdot q(b)
$$

and those of division $B$ to

$$
E\left[\pi_{B} \mid \mathbf{e}\right]=P_{B} \cdot h(b)+P_{A} \cdot q(a) .
$$

Following Choe and Ishiguro (2012), I assume that a party receives private benefits of control from having decision authority over a division, which 
is parameterized by $\lambda \in(0,1)$. Hence, $i$ 's $(i=A, B, M)$ expected payoff from private benefits of control are given by $\lambda \cdot \sum_{j=A, B} \chi_{i j} E\left[\pi_{j} \mid \mathbf{e}\right]$. Note that, according to Choe and Ishiguro (2012), the parameter $\lambda$ is used to express a party's utility from having decision authority. It is not a sharing parameter which would imply that only the remaining part of the expected specific returns goes to the owner. Contrary to Choe and Ishiguro (2012) but in line with Schmitz (2013), I assume that the owner can choose incentive contracts for the three other parties based on the contractible success of the two divisions. Let $\mathbf{w}_{A B}^{i}=\left(w_{11}^{i}, w_{10}^{i}, w_{01}^{i}, w_{00}^{i}\right)$ denote the wage schedule that owner $O$ offers to player $i(i=A, B, M)$ where $w_{11}^{i}\left(w_{00}^{i}\right)$ represents the payment to $i$ if both divisions succeed (fail), $w_{10}^{i}$ the payment if division $A$ succeeds and division $B$ fails, and $w_{01}^{i}$ the payment if division $A$ fails and division $B$ succeeds. Finally, I assume that player $i(i=A, B, M)$ is protected by limited liability in terms of $w_{11}^{i}, w_{10}^{i}, w_{01}^{i}, w_{00}^{i} \geq 0$, and that his reservation value is standardized to zero.

To summarize, manager $M$ maximizes expected utility

$$
\begin{aligned}
E U_{M}\left(e_{M} \mid e_{A}, e_{B}\right) & =P_{A} P_{B} w_{11}^{M}+P_{A}\left(1-P_{B}\right) w_{10}^{M}+\left(1-P_{A}\right) P_{B} w_{01}^{M} \\
& +\left(1-P_{A}\right)\left(1-P_{B}\right) w_{00}^{M}+\lambda \cdot \sum_{j=A, B} \chi_{M j} E\left[\pi_{j} \mid \mathbf{e}\right]-k \cdot e_{M},
\end{aligned}
$$

and division head $A$

$$
\begin{aligned}
E U_{A}\left(e_{A} \mid e_{M}, e_{B}\right) & =P_{A} P_{B} w_{11}^{A}+P_{A}\left(1-P_{B}\right) w_{10}^{A}+\left(1-P_{A}\right) P_{B} w_{01}^{A} \\
& +\left(1-P_{A}\right)\left(1-P_{B}\right) w_{00}^{A}+\lambda \cdot \sum_{j=A, B} \chi_{A j} E\left[\pi_{j} \mid \mathbf{e}\right]-c \cdot e_{A} .
\end{aligned}
$$

The objective function of $B$, denoted by $E U_{B}\left(e_{B} \mid e_{M}, e_{A}\right)$, is derived analogously to $E U_{A}\left(e_{A} \mid e_{M}, e_{B}\right)$. Owner $O$ maximizes expected profits

$$
\begin{aligned}
\pi= & \sum_{j=A, B} E\left[\pi_{j} \mid \mathbf{e}\right]+P_{A} P_{B}\left(2 R-\sum_{i=A, B, M} w_{11}^{i}\right) \\
& +P_{A}\left(1-P_{B}\right)\left(R-\sum_{i=A, B, M} w_{10}^{i}\right)+\left(1-P_{A}\right) P_{B}\left(R-\sum_{i=A, B, M} w_{01}^{i}\right) \\
& -\left(1-P_{A}\right)\left(1-P_{B}\right) \sum_{i=A, B, M} w_{00}^{i} .
\end{aligned}
$$


I follow Laffont and Martimort (2002, p. 155) and Schmitz (2005a, p. 322; 2013 , p. 110), among many others, and assume that the basic return $R$ is sufficiently large so that $O$ always wants to implement high efforts $e_{A}=e_{B}=$ $e_{M}=1 .^{1}$

The timing of events is the following: First, owner $O$ chooses an allocation of decision rights, $D$, and offers contracts $\mathbf{w}_{A B}^{i}(i=A, B, M)$ to the three other parties. Thereafter, $A, B$ and $M$ decide whether to accept or reject the respective contract. If they accept, they will simultaneously choose efforts $e_{i}(i=A, B, M)$ and decisions $(a, b)$ to maximize their objective functions. Finally, nature decides on the success of the two divisions and payoffs are realized.

\section{Optimal Contracts}

At any stage of the game, all players know that, for given $D$, the authorized decision makers will choose $(a, b)$ to maximize their respective objective functions. These decisions are anticipated by owner $O$ at the beginning of the game. Since he always wants to implement high efforts, we can directly solve for the optimal contracts $\mathbf{w}_{A B}^{i *}(i=A, B, M)$ that implement $e_{i}=1$ at lowest expected labor costs for any given allocation of authority. Note that due to the limited-liability constraints, which guarantee non-negative wages, we can ignore the participation constraints of players $A, B$ and $M$ : since each player has a zero reservation value and zero cost from choosing zero effort each feasible contract that satisfies the limited-liability constraints will be accepted.

In the following, we have to look for those contracts under which $e_{A}=$ $e_{B}=e_{M}=1$ is a Nash equilibrium. Manager $M$ 's expected utility for $e_{A}=e_{B}=e_{M}=1$ is given by $E U_{M}(1 \mid 1,1)$. If $M$ deviates to $e_{M}=0$, his

\footnotetext{
${ }^{1}$ By this simplifying assumption we can skip the analysis of all the remaining effort combinations, which would lead to many additional computations without leading to really new insights.
} 
expected utility will be $E U_{M}(0 \mid 1,1)$. Hence, $M$ will not deviate from high effort if $E U_{M}(1 \mid 1,1) \geq E U_{M}(0 \mid 1,1)$. Similarly, we must have $E U_{A}(1 \mid 1,1) \geq$ $E U_{A}(0 \mid 1,1)$ and $E U_{B}(1 \mid 1,1) \geq E U_{B}(0 \mid 1,1)$ so that players $A$ and $B$ do not deviate from high effort either. Altogether, owner $O$ minimizes expected labor costs for implementing high efforts $e_{i}=1$ subject to the three Nash equilibrium conditions $E U_{i}(1 \mid 1,1) \geq E U_{i}(0 \mid 1,1)(i=A, B, M)$. The corresponding wages describe the optimal contracts $\mathbf{w}_{A B}^{i *}=\left(w_{11}^{i *}, w_{10}^{i *}, w_{01}^{i *}, w_{00}^{i *}\right)$ $(i=A, B, M)$ that are chosen by $O$ at the first stage of the game:

Proposition 1 Let $\Lambda_{M}:=\lambda\left[\chi_{M A}(h(a)+q(b))+\chi_{M B}(h(b)+q(a))\right]$, $\Lambda_{A}:=\lambda\left[\chi_{A A} h(a)+\chi_{A B} q(a)\right]$, and $\Lambda_{B}:=\lambda\left[\chi_{B A} q(b)+\chi_{B B} h(b)\right]$.

(a) If $\Lambda_{M} \geq k / \rho$, then contract $\mathbf{w}_{A B}^{M *}=(0,0,0,0)$ is optimal; otherwise, $O$ optimally chooses $\mathbf{w}_{A B}^{M *}=\left(w_{11}^{M *}, 0,0,0\right)$ with

$$
w_{11}^{M *}=\frac{k}{3 \rho^{2}}-\frac{\Lambda_{M}}{3 \rho} .
$$

(b) If $\Lambda_{A} \geq c / \rho$, then contract $\mathbf{w}_{A B}^{A *}=(0,0,0,0)$ is optimal; otherwise $O$ optimally chooses $\mathbf{w}_{A B}^{A *}=\left(w_{11}^{A *}, w_{10}^{A *}, 0,0\right)$ with

$$
2 \rho w_{11}^{A *}+(1-2 \rho) w_{10}^{A *}=\frac{c}{\rho}-\Lambda_{A} .
$$

(c) If $\Lambda_{B} \geq c / \rho$, then contract $\mathbf{w}_{A B}^{B *}=(0,0,0,0)$ is optimal; otherwise $O$ optimally chooses $\mathbf{w}_{A B}^{B *}=\left(w_{11}^{B *}, 0, w_{01}^{B *}, 0\right)$ with

$$
2 \rho w_{11}^{B *}+(1-2 \rho) w_{01}^{B *}=\frac{c}{\rho}-\Lambda_{B} .
$$

Proof. See the Appendix.

Whether owner $O$ induces incentives for $A, B$ and $M$ by offering positive wages in case of success, crucially depends on the magnitude of the already existing incentives based on private benefits of control, described by $\rho \Lambda_{i}$ ( $i=A, B, M)$. Hence, if $\rho \Lambda_{i}$ exceeds player $i$ 's additional effort costs for choosing high instead of low effort, then $i$ is sufficiently motivated without 
any additional wage premium so that $O$ optimally saves labor costs by offering zero wages for any event. However, if incentives based on private benefits of control are not large enough - in particular, if a player has not received any authority - then owner $O$ must counterbalance missing motivation by offering sufficiently large wage premiums. In that situation, the optimal contract for manager $M$ is unique: since $M$ generates the same positive externalities for both divisions he will only obtain a positive wage if both divisions are successful. If division heads $A$ and $B$ have to be incentivized via wages, the respectively optimal contract will not be unique. Since player $i(i=A, B)$ influences both the success of his own division and overall firm success it is rational to pay him a positive wage under either event. As is shown by the right-hand sides of (2), (3) and (4), for each of the three players $A, B$ and $M$ it holds that the larger the already existing incentives based on private benefits of control the lower will be optimal expected wages because both kinds of incentives are direct substitutes in the Nash equilibrium conditions.

The results of Proposition 1 imply that if owner $O$ has to offer positive wages, he can restrict his choice of optimal contracts to those with $w_{11}^{i *}>0$ and $w_{10}^{i *}=w_{01}^{i *}=w_{00}^{i *}=0(i=A, B, M)$. This observation is also intuitively plausible. Since the given firm is characterized by strong externalities via $M$ 's effort choice and the decisions $(a, b)$, it seems reasonable to compensate the relevant parties $A, B$ and $M$ on the basis of overall firm success:

Corollary 1 If the owner has to induce positive incentives via wages, contracts based on overall firm performance with

$$
w_{11}^{M *}=\frac{k}{3 \rho^{2}}-\frac{\Lambda_{M}}{3 \rho} \quad \text { and } \quad w_{11}^{j *}=\frac{c}{2 \rho^{2}}-\frac{\Lambda_{j}}{2 \rho} \quad(j=A, B)
$$

and $w_{10}^{i *}=w_{01}^{i *}=w_{00}^{i *}=0(i=A, B, M)$ at least weakly dominate all other contracts.

The result of Corollary 1 highlights an important difference to Choe and Ishiguro (2012). In their paper, Choe and Ishiguro consider two types of incentives. First, players have exogenous incentives based on private benefits of control. This assumption is identical to the one used in my paper. 
The second kind of incentives in Choe and Ishiguro (2012) stems from intrinsic motivation of the players, which is also exogenously given. Intrinsic motivation of division heads $A$ and $B$ depends on the success of their respective division, but the intrinsic motivation of manager $M$, who contributes to the success of both divisions, depends on overall firm success (see Choe and Ishiguro 2012, p. 492). In my paper, this second kind of incentives intrinsic motivation - is replaced by optimal endogenous incentives based on contracts. Since I assume limited liability, which becomes binding under the optimal contracts, players receive positive rents in this paper as well as in Choe and Ishiguro (2012). However, the crucial difference between both settings is that the allocation of decision rights can be used in Choe and Ishiguro (2012) to align the interests of at most one division head with the owner's interests (and that of manager $M$ ), whereas in my paper optimal contracts lead to aligned interests of all four parties $O, M, A$ and $B$. In the following, I will use the optimal contracts to solve for the equilibrium allocation of authority and the corresponding organizational structures.

\section{Allocation of Decision Authority}

At the first stage of the game, $O$ has to decide on $D=\left\{\chi_{M j}, \chi_{A j}, \chi_{B j}\right\}_{j=A, B}$, which allocates decision authority over $a$ and $b$ among the players $A, B$ and $M$. In principle, there are $3^{2}=9$ possible allocations. However, since the two divisions as well as their division heads $A$ and $B$ are identical we can skip three allocations without restricting the scope of the analysis. The remaining allocations and their corresponding organizational structures, as suggested 
by Choe and Ishiguro (2012), are summarized in the following table: ${ }^{2}$

\begin{tabular}{c|c|c}
$\begin{array}{c}\text { authority } \\
\text { over } a\end{array}$ & $\begin{array}{c}\text { authority } \\
\text { over } b\end{array}$ & \begin{tabular}{c} 
organizational structure \\
\hline$M$
\end{tabular} \\
\hline$A$ & $A$ & centralization $(C)$ \\
$M$ & $A$ & concentrated delegation $(C D)$ \\
$A$ & $M$ & partial delegation $(P D)$ \\
$A$ & $B$ & decentralization $(D)$ \\
$B$ & $A$ & cross-authority delegation $(C A)$
\end{tabular}

The first (second) column contains the player that receives authority over $a$ (b) and the third column shows the corresponding organizational structure with the respective abbreviation in parentheses. A centralized organizational structure $(C)$ arises if the hierarchically highest decision maker, manager $M$, receives both decision rights. Choe and Ishiguro (2012) define an organizational structure as concentrated delegation $(C D)$ if both decision rights are allocated to a single division head. We have a three-tier hierarchy, called hierarchical delegation $(H D)$, if manager $M$ has decision authority over division $A$, and division head $A$ has decision authority over division $B$. Partial delegation $(P D)$ is given if division head $A$ has authority over his own division but manager $M$ decides on division $B$. There is decentralization $(D)$ if each division head decides on his own division. Finally, it is possible that each division head has decision authority over the other division, respectively, which is called cross-authority delegation $(C A)$.

\subsection{Centralization}

Under centralization, we have $\chi_{M A}=\chi_{M B}=1$ whereas the other indicator variables are zero. According to his objective function (5), for given wages, manager $M$ will choose $a, b=\hat{S}$ if $h>q$, and $a, b=\hat{C}$ if $h<q$. From

\footnotetext{
${ }^{2}$ The combination " $B, B$ " is skipped since it is similar to " $A, A$ ". In addition, I skip " $M, B$ " and " $B, M$ " because they are similar to " $M, A$ " and " $A, M "$.
} 
Proposition 1, we know that his compensation will be

$$
w_{11}^{M *}=\left\{\begin{array}{cll}
0 & \text { if } h>q \text { and } 2 \lambda h \geq \frac{k}{\rho} \\
0 & \text { if } h<q \text { and } 2 \lambda q \geq \frac{k}{\rho} \\
\frac{k}{3 \rho^{2}}-\frac{2 \lambda h}{3 \rho} & \text { if } h>q \text { and } 2 \lambda h<\frac{k}{\rho} \\
\frac{k}{3 \rho^{2}}-\frac{2 \lambda q}{3 \rho} & \text { if } h<q \text { and } 2 \lambda q<\frac{k}{\rho}
\end{array}\right.
$$

Since the two division heads have zero authority, they must be fully compensated via explicit incentive pay. From Corollary 1 we obtain $w_{11}^{A *}=\frac{c}{2 \rho^{2}}$ and $w_{11}^{B *}=\frac{c}{2 \rho^{2}}$. According to (1), owner $O$ 's expected profits with a centralized organization are

$$
\pi^{C}=\left\{\begin{array}{ccc}
4(R \rho+h \rho-c) & \text { if } h>q \text { and } 2 \lambda h \geq \frac{k}{\rho} \\
4(R \rho+q \rho-c) & \text { if } h<q \text { and } 2 \lambda q \geq \frac{k}{\rho} \\
4 R \rho+\left(4+\frac{8}{3} \lambda\right) h \rho-4 c-\frac{4}{3} k & \text { if } h>q \text { and } 2 \lambda h<\frac{k}{\rho} \\
4 R \rho+\left(4+\frac{8}{3} \lambda\right) q \rho-4 c-\frac{4}{3} k & \text { if } h<q \text { and } 2 \lambda q<\frac{k}{\rho}
\end{array}\right.
$$

\subsection{Concentrated Delegation}

Now division head $A$ has full decision authority with $\chi_{A A}=\chi_{A B}=1$. From (8) it follows that, for given wages, he will choose $a, b=\hat{S}$ if $h>q$, and $a, b=\hat{C}$ if $h<q$. His compensation is therefore

$$
w_{11}^{A *}=\left\{\begin{array}{cll}
0 & \text { if } h>q \text { and } \lambda h \geq \frac{c}{\rho} \\
0 & \text { if } h<q \text { and } \lambda q \geq \frac{c}{\rho} \\
\frac{c}{2 \rho^{2}}-\frac{\lambda h}{2 \rho} & \text { if } h>q \text { and } \lambda h<\frac{c}{\rho} \\
\frac{c}{2 \rho^{2}}-\frac{\lambda q}{2 \rho} & \text { if } h<q \text { and } \lambda q<\frac{c}{\rho}
\end{array}\right.
$$

Players $B$ and $M$ do not have any authority and must be fully compensated via $w_{11}^{B *}>0$ and $w_{11}^{M *}>0$. Corollary 1 yields $w_{11}^{B *}=\frac{c}{2 \rho^{2}}$ and $w_{11}^{M *}=\frac{k}{3 \rho^{2}}$. Owner $O$ 's expected profits are

$$
\pi^{C D}=\left\{\begin{array}{ccc}
4 R \rho+4 h \rho-2 c-\frac{4}{3} k & \text { if } h>q \text { and } \lambda h \geq \frac{c}{\rho} \\
4 R \rho+4 q \rho-2 c-\frac{4}{3} k & \text { if } h<q \text { and } \lambda q \geq \frac{c}{\rho} \\
4 R \rho+(4+2 \lambda) h \rho-4 c-\frac{4}{3} k & \text { if } h>q \text { and } \lambda h<\frac{c}{\rho} \\
4 R \rho+(4+2 \lambda) q \rho-4 c-\frac{4}{3} k & \text { if } h<q \text { and } \lambda q<\frac{c}{\rho} .
\end{array}\right.
$$




\subsection{Hierarchical delegation}

Since $M$ has authority over division $A$ (i.e., $\chi_{M A}=1$ ) he chooses $a=\hat{S}$ to maximize (5), implying $h(a)=h$ and $q(a)=0$. Player $A$ has authority over division $B$ (that is, $\chi_{A B}=1$ ). According to (8), for given wages, he chooses $b=\hat{S}$, implying $h(b)=h$ and $q(b)=0$. Hence, $M$ 's compensation is given by

$$
w_{11}^{M *}=\left\{\begin{array}{cll}
0 & \text { if } \quad \lambda h \geq \frac{k}{\rho} \\
\frac{k}{3 \rho^{2}}-\frac{\lambda h}{3 \rho} & \text { if } \quad \lambda h<\frac{k}{\rho},
\end{array}\right.
$$

whereas the two division heads receive wages $w_{11}^{A *}=w_{11}^{B *}=\frac{c}{2 \rho^{2}} \cdot{ }^{3}$ Owner $O$ 's expected profits can be written as

$$
\pi^{H D}=\left\{\begin{array}{clc}
4(R \rho+h \rho-c) & \text { if } & \lambda h \geq \frac{k}{\rho} \\
4 R \rho+\left(4+\frac{4}{3} \lambda\right) h \rho-4 c-\frac{4}{3} k & \text { if } & \lambda h<\frac{k}{\rho} .
\end{array}\right.
$$

\subsection{Partial Delegation}

Partial delegation is characterized by $\chi_{A A}=\chi_{M B}=1$. (5) and (8) show that $M$ optimally chooses $b=\hat{S}$, which implies $h(b)=h$ and $q(b)=0$, and $A$ chooses $a=\hat{S}$, implying $h(a)=h$ and $q(a)=0$. The corresponding wages are therefore

$$
w_{11}^{M *}=\left\{\begin{array}{cl}
0 & \text { if } \quad \lambda h \geq \frac{k}{\rho} \\
\frac{k}{3 \rho^{2}}-\frac{\lambda h}{3 \rho} & \text { if } \quad \lambda h<\frac{k}{\rho}
\end{array} \quad \text { and } \quad w_{11}^{A *}=\left\{\begin{array}{cl}
0 & \text { if } \quad \lambda h \geq \frac{c}{\rho} \\
\frac{c}{2 \rho^{2}}-\frac{\lambda h}{2 \rho} & \text { if } \quad \lambda h<\frac{c}{\rho},
\end{array}\right.\right.
$$

whereas $B$ is offered wage $w_{11}^{B *}=\frac{c}{2 \rho^{2}}$. Owner $O$ 's expected profits crucially depend on the relation of $M$ 's and $A$ 's effort costs. If $k>c$, then

$$
\pi_{k>c}^{P D}=\left\{\begin{array}{clc}
4 R \rho+\left(4+\frac{10}{3} \lambda\right) h \rho-4 c-\frac{4}{3} k & \text { if } \quad \lambda h<\frac{c}{\rho} \\
4 R \rho+\left(4+\frac{4}{3} \lambda\right) h \rho-2 c-\frac{4}{3} k & \text { if } \quad \frac{c}{\rho} \leq \lambda h<\frac{k}{\rho} \\
4 R \rho+4 h \rho-2 c & \text { if } \quad \frac{k}{\rho} \leq \lambda h,
\end{array}\right.
$$

\footnotetext{
${ }^{3}$ Note that $A$ has authority over division $B$ (i.e., $\chi_{A B}=1$ ), but $q(a)=0$ so that player $A$ does not have incentives from delegated authority.
} 
but if $k<c$, then

$$
\pi_{k<c}^{P D}=\left\{\begin{array}{ccc}
4 R \rho+\left(4+\frac{10}{3} \lambda\right) h \rho-4 c-\frac{4}{3} k & \text { if } & \lambda h<\frac{k}{\rho} \\
4 R \rho+(4+2 \lambda) h \rho-4 c & \text { if } & \frac{k}{\rho} \leq \lambda h<\frac{c}{\rho} \\
4 R \rho+4 h \rho-2 c & \text { if } \quad \frac{c}{\rho} \leq \lambda h .
\end{array}\right.
$$

\subsection{Decentralization}

Not surprisingly, in case of decentralization $\left(\chi_{A A}=\chi_{B B}=1\right)$, both division heads behave selfishly: $a=b=\hat{S}$, which implies $h(a)=h(b)=h$ and $q(a)=q(b)=0$. The division heads' wages are thus

$$
w_{11}^{A *}=w_{11}^{B *}=\left\{\begin{array}{cl}
0 & \text { if } \quad \lambda h \geq \frac{c}{\rho} \\
\frac{c}{2 \rho^{2}}-\frac{\lambda h}{2 \rho} & \text { if } \quad \lambda h<\frac{c}{\rho},
\end{array}\right.
$$

and manager $M$ obtains $w_{11}^{M *}=\frac{k}{3 \rho^{2}}$, leading to expected profits

$$
\pi^{D}=\left\{\begin{array}{cc}
4 R \rho+4 h \rho-\frac{4}{3} k & \text { if } \quad \lambda h \geq \frac{c}{\rho} \\
4 R \rho+(4+4 \lambda) h \rho-4 c-\frac{4}{3} k & \text { if } \quad \lambda h<\frac{c}{\rho},
\end{array}\right.
$$

for owner $O$.

\subsection{Cross-Authority Delegation}

This final allocation of decision rights with $\chi_{B A}=\chi_{A B}=1$ is just the opposite of decentralization. Consequently, both division heads prefer cooperative behavior $a=b=\hat{C}$, implying $q(a)=q(b)=q$ and $h(a)=h(b)=0$. Hence, we only have to replace " $h$ " by " $q$ " in $\pi^{D}$ to obtain $O$ 's expected profits for cross-authority delegation:

$$
\pi^{C A}=\left\{\begin{array}{cc}
4 R \rho+4 q \rho-\frac{4}{3} k & \text { if } \quad \lambda q \geq \frac{c}{\rho} \\
4 R \rho+(4+4 \lambda) q \rho-4 c-\frac{4}{3} k & \text { if } \quad \lambda q<\frac{c}{\rho},
\end{array}\right.
$$

\section{Optimal Organizational Structure}

In this section, I compare the expected profits of the different organizational structures to find out which of them will arise under what parameter constellations in equilibrium. An immediate observation leads to the first result: 
Proposition 2 Concentrated delegation and hierarchical delegation are never chosen by the owner.

Proof. Comparing $\pi^{C D}$ with $\pi^{D}$ shows that, if $q<h$, then decentralization dominates concentrated delegation. For $q>h$, however, the comparison of $\pi^{C D}$ and $\pi^{C A}$ shows that cross-authority delegation dominates concentrated delegation. Comparing $\pi^{H D}$ with $\pi_{k>c}^{P D}$ and $\pi_{k<c}^{P D}$ immediately shows that partial delegation always dominates hierarchical delegation, irrespective of whether $k>c$ or $k<c$.

The comparison of profits points to the following comparative disadvantages of concentrated delegation: if incentives based on private benefits of control are sufficiently large so that $O$ can save explicit incentive pay when implementing high efforts, other organizational structures will lead to the same expected returns at lower implementation costs. In particular, decentralization or cross-authority delegation, respectively, require zero wage costs for both division heads to implement high effort in this situation, whereas under concentrated delegation $O$ can only save explicit labor costs from one division head. If private benefits of control are not large enough to replace explicit incentive pay, concentrated delegation is less effective than decentralization or cross-authority delegation since it requires the same implementation costs but yields less expected returns.

Hierarchical delegation is also not optimal in economizing on implementation costs. The comparison with partial delegation shows that, under both organizational structures, $O$ can save implementation costs for the manager $M$ if private benefits of control are sufficiently large, but partial delegation additionally saves costs from one of the division heads. If private benefits of control are only moderate or rather small so that $O$ either saves implementation costs only for $M$ or no costs at all under both organizational structures, then partial delegation anyhow yields higher expected returns at identical implementation costs compared to hierarchical delegation.

Comparing the findings of Proposition 2 with the results in Choe and Ishiguro (2012) shows that, in their setting, concentrated delegation is never 
optimal either. However, concerning hierarchical delegation, my findings are in stark contrast to those in Choe and Ishiguro (2012). They show that, if $q$ is large, both decisions, $a$ and $b$, will be cooperative and lead to higher profits the larger $q$. Consequently, hierarchical delegation can be optimal in situations where the specific returns from cooperative behavior (i.e., $q$ ) considerably exceed specific returns from selfish behavior (i.e., $h$ ). Choe and Ishiguro conclude that "hierarchical delegation can emerge as an optimal organizational form when both coordination and motivation are important" (Choe and Ishiguro 2012, p. 491).

In my setting with endogenous incentives and optimal contracts, however, decisions are always selfish under hierarchical delegation. The objective functions (5) and (8) show that, under $\chi_{M A}=\chi_{A B}=1$ both decision makers $M$ and $A$ prefer selfish behavior to maximize their private benefits of control. Proposition 1 then points to a fundamental incentive problem that arises under hierarchical delegation. Under any organizational structure, owner $O$ profits from implicit incentives based on private benefits of control because they replace explicit wage premiums and, thus, reduce $O$ 's implementation costs for high effort. Under hierarchical delegation, $A$ 's implicit incentives from decision authority are given by

$$
\rho \Lambda_{A}=\rho \lambda\left[\chi_{A A} h(a)+\chi_{A B} q(a)\right]
$$

Since $\chi_{M A}=\chi_{A B}=1$ whereas all other indicator variables are zero and since under purely selfish behavior we obtain $h(a)=h>q(a)=0$, implicit incentives of division head $A$ are $\rho \Lambda_{A}=0$-despite delegation. This result is not surprising if we look at $A$ 's incentive constraint (9). Of course, authority over division $B$ is beneficial for $A$ since it leads to the expected extra utility $2 \rho \lambda\left[\chi_{A B}(h(b)+q(a))\right]=2 \rho \lambda h(b)$ in his objective function (see (8)). However, from $O$ 's perspective this fact has no value at all, since $A$ 's participation constraint is satisfied anyway and, most importantly, the incentive constraint is independent of $2 \rho \lambda h(b)$ because division head $A$ cannot influence the success of division $B$. In other words, division head $A$ may obtain the private benefits of control, $\lambda h(b)$, with positive probability, but this probability will 
be $P_{B}\left(e_{B}, e_{M}\right)=2 \rho$ irrespective of whether $A$ chooses $e_{A}=1$ or $e_{A}=0$.

Note that this result is also related to the incentive intensity principle of Milgrom and Roberts (1992). According to this principle, the "intensity of incentives should increase with the marginal productivity of effort and with the agent's ability to respond to incentives" (Milgrom and Roberts 1992, p. 599). In this model, hierarchical delegation does not work well from an incentive perspective since division head $A$ gets authority over division $B$ but this delegation does not create implicit incentives since $A$ is not able to respond with his effort choice to the owner's allocation of decision rights.

The remaining four alternatives - centralization $(C)$, partial delegation $(P D)$, decentralization $(D)$ and cross-authority delegation $(C A)$ - can be optimal organizational structures for certain parameter constellations. If the division heads are less efficient than the manager, we will obtain the following result:

Proposition 3 Let $c>k$. If the specific returns to selfish behavior exceed the specific returns to cooperative behavior (i.e., $h>q$ ), then decentralization is the optimal organizational structure; otherwise (i.e., $h<q$ ), cross-authority delegation is optimal.

Proof. See the Appendix.

The intuition for the result of Proposition 3 is the following. Recall that the compensation of $A, B$ and $M$ for exerting high effort consists of private benefits of control - which are for free for owner $O$ - and of explicit wage payments - which directly increase $O$ 's labor costs. The higher a player's private benefits of control the lower are $O$ 's labor costs for this player and vice versa. If the two division heads are less efficient than the manager (i.e., $c>k$ ), it will be profitable for $O$ to avoid explicit wage payments to the division heads by giving decision authority and, hence, private benefits of control to them. Decentralization and cross-authority delegation are the only remaining organizational structures where the decision rights are completely allocated to the two division heads. As a consequence, $O$ can reduce his labor 
costs considerably under these two organizational structures since in many situations he only has to pay the rather moderate wage $w_{11}^{M *}$ to manager $M$ without paying anything else to $A$ and $B$. However, this is impossible under centralization and partial delegation, where $O$ must always offer a high wage $w_{11}^{i *}(i \in\{A, B\})$ to one of the division heads. The relation of the specific returns, $h$ and $q$, then is decisive whether $O$ profits more from decentralization or cross-authority delegation. From Sections 4.5 and 4.6, we know that division heads prefer selfish behavior under decentralization, leading to specific returns $h$, but cooperative behavior under cross-authority delegation, leading to specific returns $q$. Therefore, it is optimal for $O$ to choose decentralization if $h>q$ and cross-authority delegation otherwise.

The optimal organizational structure is less obvious for $c<k$. First, we consider the case of $h>q$ so that decentralization dominates cross-authority delegation from the owner's point of view (see Lemma 1 in the proof of Proposition 3). Comparing $O$ 's expected profits under the organizational structures $C, P D$ and $D$ leads to the following results:

Proposition 4 Let $c<k$ and $h>q$. If $c>\frac{2}{3} k$, then decentralization will be optimal. If $c<\frac{2}{3} k$ and $h \geq \bar{h}:=\frac{k}{\lambda \rho}$, then partial delegation will be optimal. If $h<\bar{h}$ and $c$ is sufficiently small, then centralization will be optimal.

Proof. See the Appendix.

Proposition 4 deals with the situation in which the division heads are more efficient than the manager and specific returns to selfish behavior exceed the specific returns to cooperative behavior. The proposition states sufficient conditions for decentralization, partial delegation and centralization being optimal. A complete pairwise comparison of expected profits for the case $h>q$ leads to the findings that are summarized in Figure $1 .^{4}$

Proposition 4 and Figure 1 show that owner $O$ will choose decentralization as optimal organizational structure if the division heads' effort costs are

\footnotetext{
${ }^{4}$ For the construction of Figure 1 see the Appendix. Note that, in Figure 1, $\frac{2}{3} \lambda q \rho<$ $\frac{k}{3}<\lambda q \rho<\frac{k}{2}<\frac{2}{3} k$, but other relations are also possible.
} 


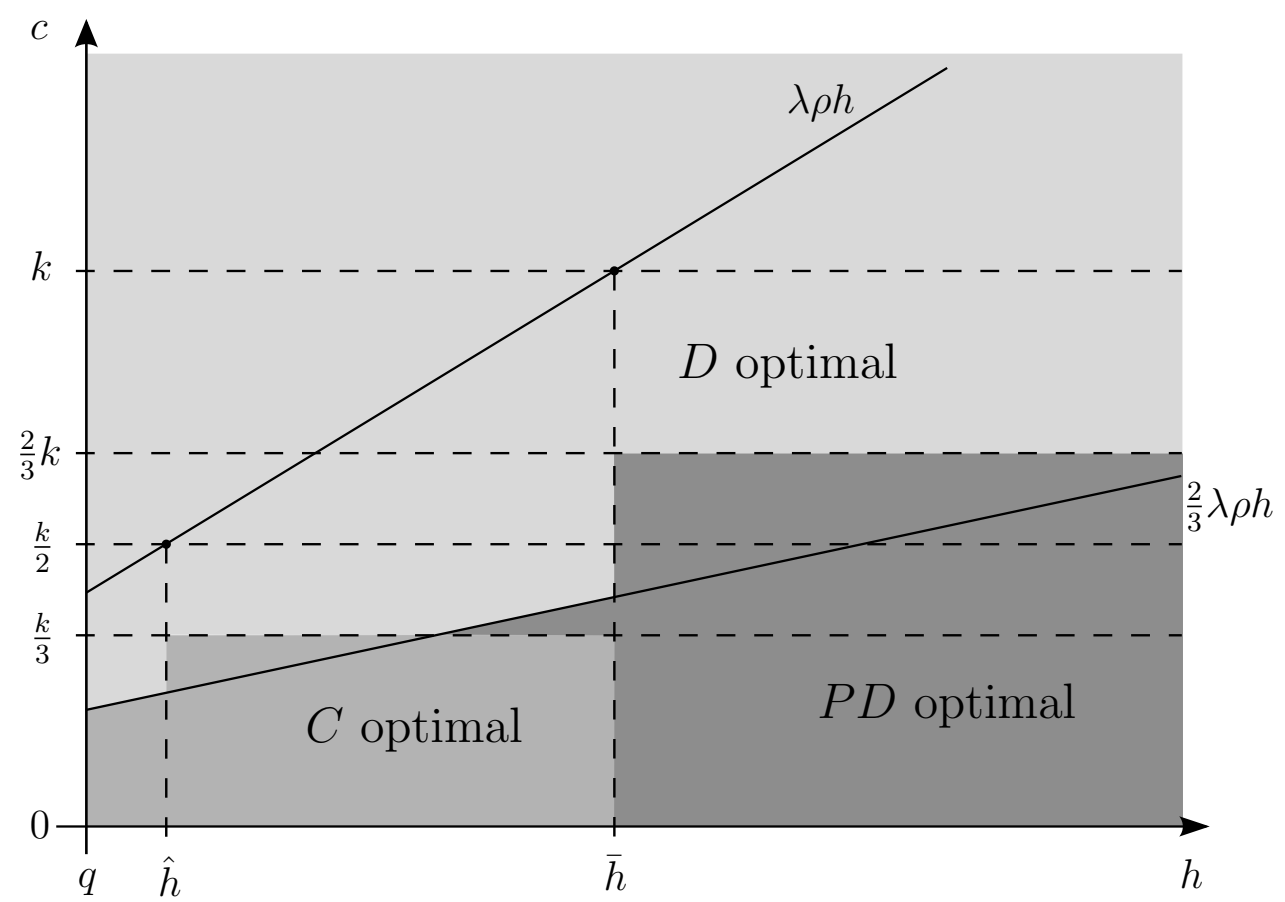

Figure 1: Optimal organizational structure for $h>q$

sufficiently large. The intuition for this result has been given above within the discussion of Proposition 3: decentralization is optimal since both division heads obtain incentives via private benefits of control, which leads to a substantial saving of labor costs for $O$ compared to the structures $C$ and $P D$. Under these structures, at most one of the division heads receives private benefits of control. Consequently, $C$ and $P D$ may only be optimal if $c<k$. Figure 1 shows that for $C$ to be optimal $c$ even has to be smaller than $\frac{k}{3}$, whereas $P D$ can only be optimal if $c$ does not exceed $\frac{2}{3} k$.

Note that for $h>q$ both $C$ and $P D$ lead to selfish behavior so that large values of $h$ are beneficial for $O$ under either structure because specific returns increase in $h$ (see $\pi^{C}$ and $\pi_{k>c}^{P D}$ ). However, as Proposition 4 and Figure 1 point out, only $P D$ can be optimal for $h \geq \bar{h}$. This observation can be explained by the following table based on $h \geq \bar{h}$ - the relevant range in Figure 1 . In the 
table, $\Delta e l c$ denotes the expected labor costs under $P D$ minus the expected labor costs under $C$, and $\Delta e s r$ the expected specific returns under $P D$ minus the expected specific returns under $C$ :

\begin{tabular}{c|c|c}
$\lambda \rho h$ & $\Delta e l c$ & $\Delta e s r$ \\
\hline$\geq k$ & $-2 c$ & 0 \\
$\in\left[\frac{k}{2}, k\right)$ & $\frac{4}{3} k-2 c$ & $\frac{4}{3} \lambda \rho h$ \\
$\in\left[c, \frac{k}{2}\right)$ & $-2 c$ & $-\frac{4}{3} \lambda \rho h$
\end{tabular}

The first row of the table shows that $P D$ clearly dominates $C$ under $\lambda \rho h \geq k$, since the former structure leads to the same expected specific returns but lower labor costs than the latter one. For intermediate values - i.e., $\lambda \rho h \in$ $\left[\frac{k}{2}, k\right)$ - we have a trade-off. On the one hand, $\frac{4}{3} k-2 c>0 \Leftrightarrow \frac{2}{3} k>c$ is true in the relevant region of Figure 1, thus showing that $P D$ leads to higher labor costs than $C$. On the other hand, $P D$ is also associated with higher specific returns. As Figure 1 shows, there are parameter constellations for $\lambda \rho h \in$ $\left[\frac{k}{2}, k\right)$ where the cost disadvantage of $P D$ has a higher weight, and other constellations where the additional returns become dominant. The third row of the table deals with rather small values of $h$ with $\lambda \rho h \in\left[c, \frac{k}{2}\right)$. For Figure 1 , this means that only parameter constellations with $h<\hat{h}$ below the line $\lambda \rho h$ are relevant. Now, $P D$ has a cost advantage but a return disadvantage. $C$ will beat $P D$ if the return disadvantage becomes dominant, that is, $\frac{4}{3} \lambda \rho h>$ $2 c \Leftrightarrow \frac{2}{3} \lambda \rho h>c$, which is confirmed by Figure 1. Altogether, $P D$ dominates $C$ if $h$ is sufficiently large since it either leads to rather low labor costs or large specific returns, whereas the organizational structure $C$ becomes optimal if $h$ does nor exceed $q$ very much and costs $c$ are sufficiently small so that the division heads' effort costs are negligible and the cost advantage of $P D$ is dominated by $C$ 's advantage of higher specific returns.

According to Proposition 3, in the half of the parameter space with $h<q$, cross-authority delegation is optimal as long as the division heads are less efficient than the manager (i.e., $c>k$ ). Therefore, in the following, I consider the remaining parameter constellations with $h<q$ and $c<k$ in which either $C A$ or $P D$ or $C$ is the optimal organizational structure. The comparison of 
expected profits leads to the results of Proposition 5:

Proposition 5 Let $c<k$ and $h<q$. If $c>\frac{2}{3} k$, then cross-authority delegation will be optimal. If $c<\frac{2}{3} k$ and $h$ is sufficiently close to $q$ satisfying $h \geq \bar{h}$, then partial delegation will be optimal. If $h<\bar{h}$ and $c$ is sufficiently small, then centralization will be optimal.

Proof. See the Appendix.

Proposition 5 shows that if the specific returns to cooperative behavior become larger than those to selfish behavior, we have to replace decentralization by cross-authority delegation for sufficiently large values of $c$, but the other findings of Proposition 4 for partial delegation and centralization remain rather stable. Figure 2 summarizes the overall results from all pairwise comparisons for the case $h<q .{ }^{5}$

Figure 1 has shown that $P D$ cannot be optimal if $h$ is sufficiently small. This result is confirmed by Figure 2. Here, $h$ is so small that $\lambda \rho h<\frac{k}{3}$. As a consequence, the organizational structure $P D$ does not appear in the figure. Instead, $C A$ is optimal if $c$ is sufficiently large, otherwise $C$ is optimal. According to Lemma 1, $C A$ will always dominate $D$ if specific returns to cooperative behavior exceed the specific returns to selfish behavior (i.e., $q>$ $h)$. Hence, $D$ does not appear in Figure 2 either. If the division heads' effort costs $c$ are large, it will be optimal for owner $O$ to create incentives based on private benefits of control exclusively for division heads $A$ and $B$ to save labor costs when implementing high efforts. This aim is achieved by relying on cross-authority delegation. If, however, the manager's effort costs $k$ are large relative to those of the division heads, it will be better for $O$ to give the manager full incentives based on private benefits of control by using centralization.

Proposition 5 shows that in situations in which the specific returns to selfish behavior, $h$, are rather large - so that $\lambda \rho h \geq k \Leftrightarrow h \geq \bar{h}$ and $q-h$ is

\footnotetext{
${ }^{5}$ For the construction of Figure 2 see the Appendix. Note that, as in Figure 1, the ranking of intercepts at the vertical axis in Figure 2 is not unique.
} 


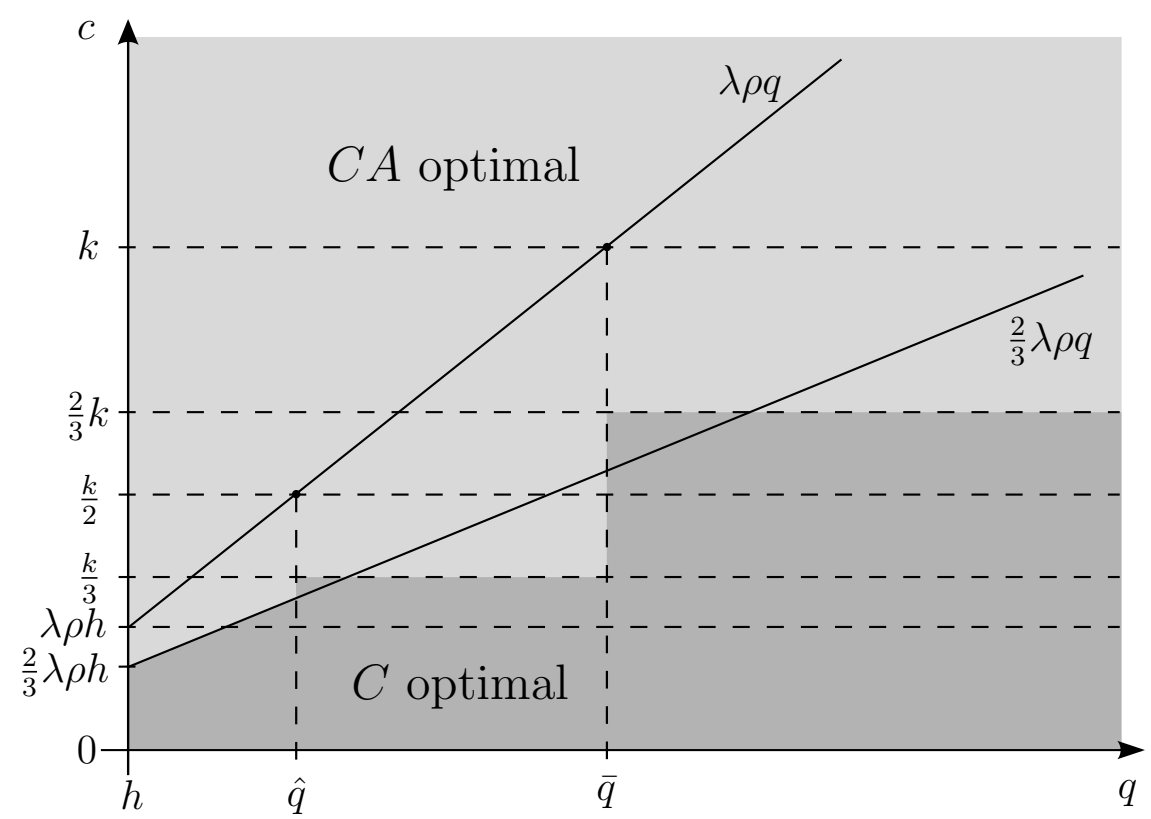

Figure 2: Optimal organizational structure for $h<q$ and $\lambda \rho h<k$

small - the organizational structure $P D$ will dominate both $C A$ and $C$ for parameter values satisfying $c<\frac{2}{3} k$. This finding is shown in Figure 3, which has $\lambda \rho h>k .{ }^{6}$ As we know from Sections 4.1 to 4.6, partial delegation (PD) and decentralization $(D)$ are the only organizational structures that always induce selfish behavior irrespective of whether $h$ exceeds $q$ or vice versa. In the given situation with large specific returns $h$ that do not differ much from $q$ an organizational structure which induces selfish behavior is quite beneficial for the owner. Since, however, $q>h$ organizational structure $D$ cannot be optimal because it is always outperformed by $C A$ which works very similar to $D$ but is based on the higher specific returns $q$. Therefore, owner $O$ relies on partial delegation $P D$. The intuition for this result can be best seen from a direct comparison of the three profits for the relevant region of the parameter

\footnotetext{
${ }^{6}$ For the construction of Figure 3 see the Appendix.
} 


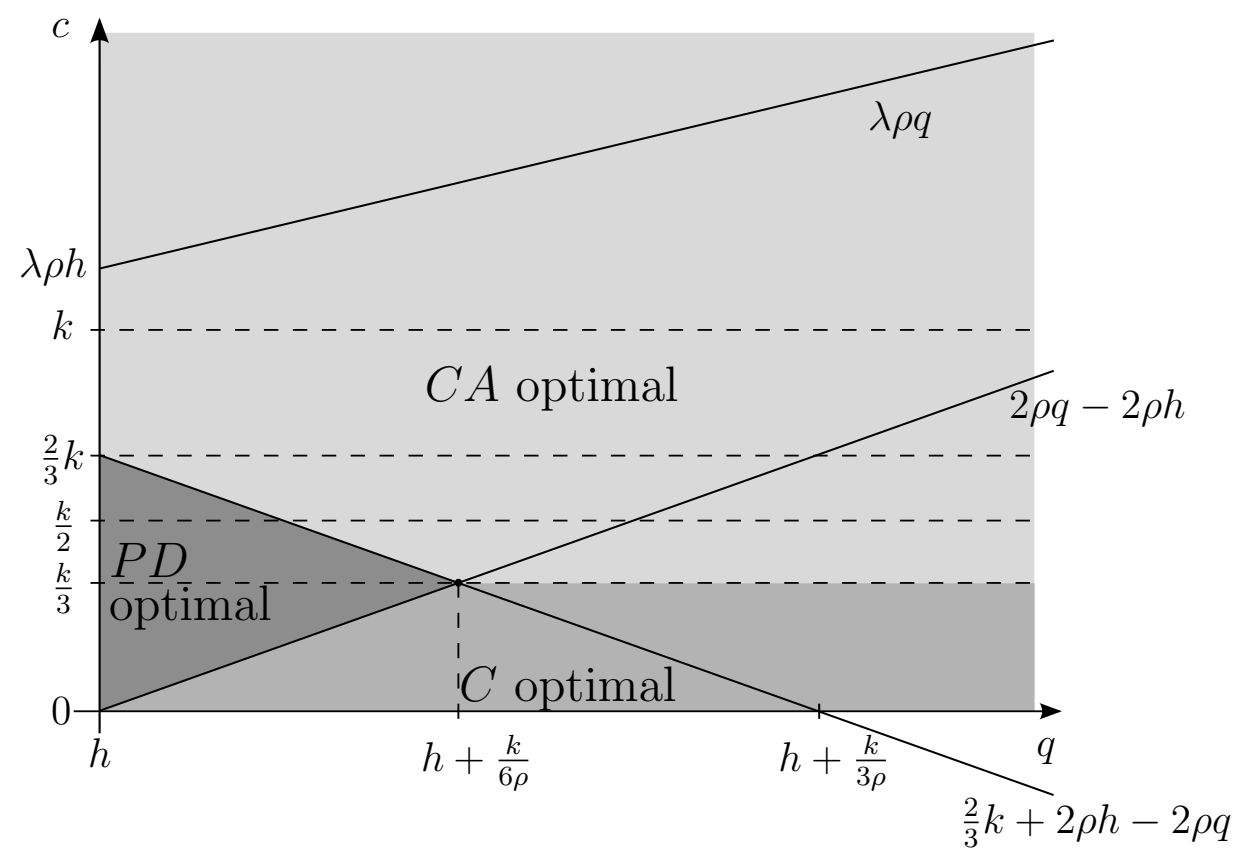

Figure 3: Optimal organizational structure for $h<q$ and $\lambda \rho h>k$

space. From 4.1, 4.4 and 4.6 we obtain

$$
\begin{aligned}
\pi^{C} & =4 R \rho+4 q \rho-4 c, \\
\pi_{k>c}^{P D} & =4 R \rho+4 h \rho-2 c \\
\text { and } \quad \pi^{C A} & =4 R \rho+4 q \rho-\frac{4}{3} k .
\end{aligned}
$$

Thus, if $h$ and $q$ do not differ much, differences in expected specific returns are negligible. However, since $h$ and $q$ are large, incentives from private benefits of control are used in each of the three organizational structures to save labor costs. The expression for $\pi^{C}$ points out that centralization works poorly as labor costs for the manager are saved but $O$ still has to pay both workers. Under partial delegation, however, $O$ saves labor costs for the manager and one division head so that he only has to pay the other division head. Finally, cross-authority delegation also works quite well since $O$ saves labor costs for both division heads and only has to pay the manager. 
Thus, the direct comparison between $P D$ and $C A$ crucially depends on the relation between the manager's and the division heads' effort costs. $P D$ has a cost advantage if savings from not paying the manager are sufficiently large: $2 c<\frac{4}{3} k \Leftrightarrow c<\frac{2}{3} k$, which is exactly the condition from the beginning of this paragraph.

\section{Conclusion}

In this paper, I analyze the optimal organizational structure of a corporation with two divisions. The corporation exhibits two kinds of positive externalities. First, the CEO's effort positively contributes to the success of both divisions. The performance of each division also depends on the effort of the respective division head, which leads to a kind of team problem in both organizational units. Second, if a division behaves cooperatively, the division's success will increase the performance of the other division.

The owner of the corporation can control the behavior of the CEO and the division heads via two instruments. On the one hand, the owner can use optimal incentive contracts based on the verifiable success of the two divisions. This instrument determines the effort choices of the CEO and the division heads. On the other hand, the owner allocates decision authority over the two divisions to the three players. The behavioral implications of this allocation are twofold: (1) the authorized player is directed towards more selfish or more cooperative behavior, (2) the allocation influences the players' private benefits of control and, thus, their incentives for choosing high efforts. The paper has shown, that the interplay of these two effects determines the optimal organizational structure.

Four different structures can be optimal - decentralization, cross-authority delegation, centralization and partial delegation. Decentralization will be optimal if selfish behavior is more important than cooperative behavior and division heads are less efficient than the CEO. If, however, cooperation is more important than selfish behavior and division heads are still less efficient than 
the CEO, then cross-authority delegation turns out to be optimal. If the CEO is less efficient than the division heads, the two remaining organizational structures can be optimal. Centralization is beneficial since the CEO, who has full decision authority, flexibly either chooses selfish or cooperative behavior, depending on which one is more effective. However, centralization has the drawback that private benefits of control can only reduce the owner's labor costs for the CEO. Partial delegation always leads to selfish behavior and, hence, is disadvantageous if cooperative behavior is considerably more important than selfish behavior. However, compared to centralization, partial delegation can reduce the labor costs for the CEO and one division head, which is beneficial from the owner's perspective.

\section{Appendix}

Proof of Proposition 1:

We have

$$
\begin{aligned}
E U_{M}(1 \mid 1,1) & =4 \rho^{2} w_{11}^{M}+2 \rho(1-2 \rho)\left(w_{10}^{M}+w_{01}^{M}\right)+(1-2 \rho)^{2} w_{00}^{M} \\
& +2 \rho \lambda\left[\chi_{M A}(h(a)+q(b))+\chi_{M B}(h(b)+q(a))\right]-k
\end{aligned}
$$

and, in case of deviation to low effort $e_{M}=0$,

$$
\begin{aligned}
E U_{M}(0 \mid 1,1) & =\rho^{2} w_{11}^{M}+\rho(1-\rho)\left(w_{10}^{M}+w_{01}^{M}\right)+(1-\rho)^{2} w_{00}^{M} \\
& +\rho \lambda\left[\chi_{M A}(h(a)+q(b))+\chi_{M B}(h(b)+q(a))\right] .
\end{aligned}
$$

Condition $E U_{M}(1 \mid 1,1) \geq E U_{M}(0 \mid 1,1)$ can therefore be written as

$$
\begin{aligned}
& 3 \rho^{2} w_{11}^{M}-\rho(3 \rho-1)\left(w_{10}^{M}+w_{01}^{M}\right)-\rho(2-3 \rho) w_{00}^{M} \\
& \quad+\rho \lambda\left[\chi_{M A}(h(a)+q(b))+\chi_{M B}(h(b)+q(a))\right] \geq k .
\end{aligned}
$$

Owner $O$ 's expected labor costs from inducing high effort to $M$ are

$$
4 \rho^{2} w_{11}^{M}+2 \rho(1-2 \rho)\left(w_{10}^{M}+w_{01}^{M}\right)+(1-2 \rho)^{2} w_{00}^{M} .
$$


There are two possibilities: if $M$ has got decision rights for $a$ and/or $b$ and his private benefits of control are sufficiently large so that $\rho \lambda\left[\chi_{M A}(h(a)+q(b))+\right.$ $\left.\chi_{M B}(h(b)+q(a))\right] \geq k$, then $M$ 's motivation is already large enough so that $O$ optimally chooses $w_{11}^{M}=w_{10}^{M}=w_{01}^{M}=w_{00}^{M}=0$ to save labor costs; otherwise, $O$ will minimize (7) subject to (6). Obviously, $w_{00}^{M}=0$ is optimal. In addition, without loss of generality, we can set $w_{01}^{M}=0$. Thus, the problem reduces to

$$
\begin{gathered}
\min _{w_{11}^{M}, w_{10}^{M}} 4 \rho^{2} w_{11}^{M}+2 \rho(1-2 \rho) w_{10}^{M} \quad \text { subject to } \\
3 \rho w_{11}^{M}-(3 \rho-1) w_{10}^{M}+\Lambda_{M} \geq \frac{k}{\rho}
\end{gathered}
$$

with $\Lambda_{M}:=\lambda\left[\chi_{M A}(h(a)+q(b))+\chi_{M B}(h(b)+q(a))\right]$. If $\rho \geq 1 / 3$, then $w_{10}^{M}=0$ and $w_{11}^{M}=\frac{k}{3 \rho^{2}}-\frac{\Lambda_{M}}{3 \rho}$ are optimal; otherwise - that is, $\rho<1 / 3-$ owner $O$ optimally chooses from the iso-cost curves with costs $C_{M}$ that are described by

$$
w_{10}^{M}=\frac{C_{M}}{2 \rho(1-2 \rho)}-\frac{2 \rho}{1-2 \rho} w_{11}^{M}
$$

the one that corresponds to the lowest possible costs $C_{M}$ and, at the same time, satisfies $M$ 's Nash equilibrium condition

$$
w_{10}^{M} \geq \frac{\frac{k}{\rho}-\Lambda_{M}}{1-3 \rho}-\frac{3 \rho}{1-3 \rho} w_{11}^{M} .
$$

Since the absolute value of the slope of the iso-cost curves is smaller than the absolute value of the slope of the Nash equilibrium condition - i.e., $\frac{2 \rho}{1-2 \rho}<$ $\frac{3 \rho}{1-3 \rho}-$ it is again optimal for $O$ to choose $w_{10}^{M}=0$ and $w_{11}^{M}=\frac{k}{3 \rho^{2}}-\frac{\Lambda_{M}}{3 \rho}$.

Given $e_{A}=e_{B}=e_{M}=1$, player $A$ 's expected utility amounts to

$$
\begin{aligned}
E U_{A}(1 \mid 1,1) & =4 \rho^{2} w_{11}^{A}+2 \rho(1-2 \rho)\left(w_{10}^{A}+w_{01}^{A}\right)+(1-2 \rho)^{2} w_{0.0}^{A} \\
& +2 \rho \lambda\left[\chi_{A A}(h(a)+q(b))+\chi_{A B}(h(b)+q(a))\right]-c
\end{aligned}
$$

Deviating to low effort leads to

$$
\begin{aligned}
E U_{A}(0 \mid 1,1) & =2 \rho^{2} w_{11}^{A}+\rho(1-2 \rho) w_{10}^{A}+2 \rho(1-\rho) w_{01}^{A}+(1-\rho)(1-2 \rho) w_{00}^{A} \\
& +\lambda\left[\chi_{A A}(\rho h(a)+2 \rho q(b))+\chi_{A B}(2 \rho h(b)+\rho q(a))\right] .
\end{aligned}
$$


Therefore, $A$ will not deviate to low effort if

$$
2 \rho^{2}\left(w_{11}^{A}-w_{01}^{A}\right)+\rho(1-2 \rho)\left(w_{10}^{A}-w_{00}^{A}\right)+\rho \lambda\left[\chi_{A A} h(a)+\chi_{A B} q(a)\right] \geq c .
$$

Since $O$ wants to minimize expected labor costs

$$
4 \rho^{2} w_{11}^{A}+2 \rho(1-2 \rho)\left(w_{10}^{A}+w_{01}^{A}\right)+(1-2 \rho)^{2} w_{00}^{A},
$$

he optimally chooses $w_{00}^{A}=w_{01}^{A}=0$. The cost minimization problem thus boils down to

$$
\begin{gathered}
\min _{w_{11}^{A}, w_{10}^{A}} 4 \rho^{2} w_{11}^{A}+2 \rho(1-2 \rho) w_{10}^{A} \quad \text { subject to } \\
2 \rho^{2} w_{11}^{A}+\rho(1-2 \rho) w_{10}^{A}+\rho \lambda\left[\chi_{A A} h(a)+\chi_{A B} q(a)\right] \geq c .
\end{gathered}
$$

Again, if private benefits of control are sufficiently large -, i.e., $\rho \lambda\left[\chi_{A A} h(a)+\right.$ $\left.\chi_{A B} q(a)\right] \geq c$ - then the choice of $w_{11}^{A}=w_{10}^{A}=0$ is optimal. Otherwise, $O$ minimizes costs by minimizing $2 \rho w_{11}^{A}+(1-2 \rho) w_{10}^{A}$ subject to $(9)$. Thus, the best $O$ can do is to choose $w_{11}^{A}$ and $w_{10}^{A}$ so that (9) becomes binding. Optimal wages $w_{11}^{A}$ and $w_{10}^{A}$ are therefore described by

$$
2 \rho w_{11}^{A}+(1-2 \rho) w_{10}^{A}=\frac{c}{\rho}-\Lambda_{A}
$$

with $\Lambda_{A}:=\lambda\left[\chi_{A A} h(a)+\chi_{A B} q(a)\right]$.

Analogous results can be found for player $B$ : optimal is always $w_{00}^{B}=$ $w_{10}^{B}=0$. If $\lambda \rho\left[\chi_{B A} q(b)+\chi_{B B} h(b)\right] \geq c$, then $w_{11}^{B}=w_{01}^{B}=0$ is optimal; otherwise optimal incentives for $B$ are described by

$$
2 \rho w_{11}^{B}+(1-2 \rho) w_{01}^{B}=\frac{c}{\rho}-\Lambda_{B}
$$

with $\Lambda_{B}=\lambda\left[\chi_{B A} q(b)+\chi_{B B} h(b)\right]$.

Proof of Proposition 3:

To prove the proposition, I start with the following useful observation:

Lemma 1 If $h>q$, then $\pi^{D}>\pi^{C A}$. If $h<q$, then $\pi^{C A}>\pi^{D}$. 
Proof. Let $h>q$. If,$\frac{c}{\rho} \leq \lambda q<\lambda h$, then $\pi^{D}>\pi^{C A} \Leftrightarrow 4 h \rho-\frac{4}{3} k>$ $4 q \rho-\frac{4}{3} k \Leftrightarrow h>q$, which is true. If $\lambda q<\frac{c}{\rho} \leq \lambda h$, then $\pi^{D}>\pi^{C A} \Leftrightarrow$ $4 h \rho-\frac{4}{3} k>(4+4 \lambda) q \rho-4 c-\frac{4}{3} k \Leftrightarrow(h-q) \rho+c-\lambda \rho q>0$, which is true since $h>q$ and $\lambda q<\frac{c}{\rho}$ by assumption. If $\lambda q<\lambda h<\frac{c}{\rho}$, then $\pi^{D}>\pi^{C A} \Leftrightarrow$ $(4+4 \lambda) h \rho-4 c-\frac{4}{3} k>(4+4 \lambda) q \rho-4 c-\frac{4}{3} k \Leftrightarrow h>q$ is true.

Now, let $h<q$. If $\frac{c}{\rho} \leq \lambda h<\lambda q$, then $\pi^{C A}>\pi^{D} \Leftrightarrow q>h$ is true. If $\lambda h<\frac{c}{\rho} \leq \lambda q$, then $\pi^{C A}>\pi^{D} \Leftrightarrow(q-h) \rho+c-\lambda \rho h>0$, which is true since $h<q$ and $\lambda h<\frac{c}{\rho}$. If $\lambda h<\lambda q<\frac{c}{\rho}$, then $\pi^{C A}>\pi^{D} \Leftrightarrow q>h$ is true.

Next, the following observation can be proved:

Lemma 2 Let $c>k$. If $h>q$, then $\pi^{D}>\pi^{C}$. If $h<q$, then $\pi^{C A}>\pi^{C}$.

Proof. Suppose $h>q$. If $\lambda h<\frac{k}{2 \rho}<\frac{c}{\rho}$, then $\pi^{D}>\pi^{C} \Leftrightarrow(4+4 \lambda) h \rho-$ $4 c-\frac{4}{3} k>\left(4+\frac{8}{3} \lambda\right) h \rho-4 c-\frac{4}{3} k \Leftrightarrow 4 \lambda>\frac{8}{3} \lambda$ is true. If $\frac{k}{2 \rho} \leq \lambda h<\frac{c}{\rho}$, then $\pi^{D}>\pi^{C} \Leftrightarrow(4+4 \lambda) h \rho-4 c-\frac{4}{3} k>4(h \rho-c) \Leftrightarrow \lambda \rho h-\frac{1}{3} k>0$, which is true since $\frac{k}{2 \rho} \leq \lambda h$. If $\frac{k}{2 \rho}<\frac{c}{\rho} \leq \lambda h$, then $\pi^{D}>\pi^{C} \Leftrightarrow 4 h \rho-\frac{4}{3} k>4(h \rho-c) \Leftrightarrow$ $\frac{1}{3} k<c$, which is true since $c>k$.

Now, suppose $h<q$. If $\lambda q<\frac{k}{2 \rho}<\frac{c}{\rho}$, then $\pi^{C A}>\pi^{C} \Leftrightarrow 4 \lambda>\frac{8}{3} \lambda$ is true. If $\frac{k}{2 \rho} \leq \lambda q<\frac{c}{\rho}$, then $\pi^{C A}>\pi^{C} \Leftrightarrow \lambda \rho q-\frac{1}{3} k>0$ is true since $\frac{k}{2 \rho} \leq \lambda q$. If $\frac{k}{2 \rho}<\frac{c}{\rho} \leq \lambda q$, then $\pi^{C A}>\pi^{C} \Leftrightarrow \frac{1}{3} k<c$ is true since $c>k$.

Finally, we have to compare $O$ 's expected profits under $D$ and $C A$ with those under $P D$, respectively:

Lemma 3 Let $c>k$. If $h>q$, then $\pi^{D}>\pi_{k<c}^{P D}$. If $h<q$, then $\pi^{C A}>\pi_{k<c}^{P D}$.

Proof. Suppose $h>q$. If $\lambda h<\frac{k}{\rho}<\frac{c}{\rho}$, then $\pi^{D}>\pi_{k<c}^{P D} \Leftrightarrow(4+4 \lambda) h \rho-$ $4 c-\frac{4}{3} k>\left(4+\frac{10}{3} \lambda\right) h \rho-4 c-\frac{4}{3} k \Leftrightarrow 4 \lambda>\frac{10}{3} \lambda$ is true. If $\frac{k}{\rho} \leq \lambda h<\frac{c}{\rho}$, then $\pi^{D}>\pi_{k<c}^{P D} \Leftrightarrow(4+4 \lambda) h \rho-4 c-\frac{4}{3} k>(4+2 \lambda) h \rho-4 c \Leftrightarrow \lambda \rho h-\frac{2}{3} k>0$ is true since $\frac{k}{\rho} \leq \lambda h$. If $\frac{k}{\rho}<\frac{c}{\rho} \leq \lambda h$, then $\pi^{D}>\pi_{k<c}^{P D} \Leftrightarrow 4 h \rho-\frac{4}{3} k>4 h \rho-2 c \Leftrightarrow$ $\frac{2}{3} k<c$ is true since $c>k$.

Now, suppose $h<q$. If $\lambda q>\lambda h \geq \frac{c}{\rho}$, then $\pi^{C A}>\pi_{k<c}^{P D} \Leftrightarrow 4 q \rho-\frac{4}{3} k>$ $4 h \rho-2 c \Leftrightarrow(q-h) \rho+\frac{1}{2} c-\frac{1}{3} k>0$ is true since $h<q$ and $c>k$. If $\frac{k}{\rho} \leq \lambda h<\lambda q<\frac{c}{\rho}$, then $\pi^{C A}>\pi_{k<c}^{P D} \Leftrightarrow(4+4 \lambda) q \rho-4 c-\frac{4}{3} k>(4+2 \lambda) h \rho-$ $4 c \Leftrightarrow(q-h)\left(\rho+\frac{1}{2} \lambda \rho\right)+\frac{1}{2} \lambda \rho q-\frac{1}{3} k>0$ is true since $h<q$ and $\frac{k}{\rho}<\lambda q$. 
If $\frac{k}{\rho} \leq \lambda h<\frac{c}{\rho} \leq \lambda q$, then $\pi^{C A}>\pi_{k<c}^{P D} \Leftrightarrow 4 q \rho-\frac{4}{3} k>(4+2 \lambda) h \rho-4 c \Leftrightarrow$ $(q-h) \rho+\frac{1}{3}(c-k)+\frac{2}{3} c-\frac{1}{2} \lambda \rho h>0$ is true since $h<q, c>k$ and $\lambda h<\frac{c}{\rho}$. If $\lambda h<\lambda q<\frac{k}{\rho}$ or $\lambda h<\frac{k}{\rho}<\lambda q<\frac{c}{\rho}$, then $\pi^{C A}>\pi_{k<c}^{P D} \Leftrightarrow(4+4 \lambda) q \rho-4 c-\frac{4}{3} k>$ $\left(4+\frac{10}{3} \lambda\right) h \rho-4 c-\frac{4}{3} k \Leftrightarrow(4+4 \lambda) q>\left(4+\frac{10}{3} \lambda\right) h$ is true since $h<q$. If $\lambda h<\frac{k}{\rho}<\frac{c}{\rho} \leq \lambda q$, then $\pi^{C A}>\pi_{k<c}^{P D} \Leftrightarrow 4 q \rho-\frac{4}{3} k>\left(4+\frac{10}{3} \lambda\right) h \rho-4 c-\frac{4}{3} k \Leftrightarrow$ $(q-h) \rho+c-\frac{5}{6} \lambda \rho h>0$ is true since $h<q$ and $\lambda h<\frac{c}{\rho}$.

Lemmas 1-3 together prove the result of Proposition 3.

\section{Proof of Proposition 4:}

The pairwise comparisons of expected profits $\pi^{D}, \pi^{C}$ and $\pi_{k>c}^{P D}$ can be summarized as follows: ${ }^{7}$ If $c>k / 2$, then $\pi^{D}>\pi^{C}$ and $\pi_{k>c}^{P D}>\pi^{C}$. In addition, we obtain

$$
\begin{aligned}
& \pi^{D}>\pi^{C} \Leftrightarrow\left\{\begin{array}{l}
\text { if } \quad c<\frac{k}{2} \leq \lambda \rho h \text { then } \frac{k}{3}<c \\
\text { if } c \leq \lambda \rho h<\frac{k}{2} \text { then } \frac{2}{3} \lambda \rho h<c \\
\text { if } \lambda \rho h<c<\frac{k}{2}
\end{array}\right. \\
& \pi^{D}>\pi_{k>c}^{P D} \Leftrightarrow\left\{\begin{array}{l}
\text { if } c<k \leq \lambda \rho h \text { then } \frac{2}{3} k<c \\
\text { if } c \leq \lambda \rho h<k \text { then } \frac{2}{3} \lambda \rho h<c \\
\text { if } \lambda \rho h<c<k
\end{array}\right. \\
& \pi_{k>c}^{P D}>\pi^{C} \Leftrightarrow\left\{\begin{array}{l}
\text { if } c<\frac{k}{2}<k \leq \lambda \rho h \\
\text { if } c<\frac{k}{2} \leq \lambda \rho h<k \text { then } \frac{2}{3}(k-\lambda \rho h)<c \\
\text { if } c \leq \lambda \rho h<\frac{k}{2}<k \text { then } \quad \frac{2}{3} \lambda \rho h<c \\
\text { if } \lambda \rho h<c<\frac{k}{2}<k
\end{array}\right.
\end{aligned}
$$

The claim on the optimality of decentralization becomes clear from $\pi^{D}>\pi^{C}$ for $c>k / 2$ and from lines 1 and 2 of the comparison $\pi^{D}>\pi_{k>c}^{P D}$, which are both satisfied for $c>\frac{2}{3} k$. Optimality of partial delegation follows from the first line of $\pi^{D}>\pi_{k>c}^{P D}$ and the first line of $\pi_{k>c}^{P D}>\pi^{C}$. Optimality of centralization can be seen from lines 1 and 2 of $\pi^{D}>\pi^{C}$ and lines 2 and 3 of $\pi_{k>c}^{P D}>\pi^{C}$.

\footnotetext{
${ }^{7}$ See the Additional Pages for the Referees.
} 


\section{Construction of Figure 1:}

From Lemma 1 in the Appendix, we know that $D$ dominates $C A$ as long as $h>q$. Thus, $C A$ will not appear in Figure 1 so that we only have to compare $D, C$ and $P D$. Proposition 3 shows that $D$ is optimal under $h>q$ if $c>k$, and the proof of Proposition 4 points out that $D$ dominates both $C$ and $P D$, if $c \in\left(\frac{2}{3} k, k\right)$ (note that $\pi^{D}>\pi_{k>c}^{P D}$ for $c \leq \lambda \rho h<k$ since $\frac{2}{3} \lambda \rho h<c$ is true under $c>\frac{2}{3} k$ because of $\lambda \rho h<k$ ). In addition, from the proof of Proposition 4, we know that $D$ is optimal under $\lambda \rho h<c$. Furthermore, $D$ is optimal if $\frac{2}{3} \lambda \rho h<c<\lambda \rho h<\frac{k}{2}$. Finally, $\pi^{D}>\pi^{C}$ under $c>k / 2$ as well as under $c<\frac{k}{2} \leq \lambda \rho h$ and $\frac{k}{3}<c$. Together with $\pi^{D}>\pi_{k>c}^{P D}$ under $c \leq \lambda \rho h<k$ and $\frac{2}{3} \lambda \rho h<c$ we obtain that $D$ is also optimal in the region $\frac{2}{3} \lambda \rho h<c<\lambda \rho h$ with $c>\frac{k}{3}$ and $\frac{k}{2} \leq \lambda \rho h<k$ (note that the last inequality can be rewritten as $\hat{h} \leq h<\bar{h}$ with $\hat{h}:=\frac{k}{2 \lambda \rho}$ and $\bar{h}=\frac{k}{\lambda \rho}$ ).

In the remainder of the parameter space either $C$ or $P D$ is the optimal organizational structure. The third line of $\pi_{k>c}^{P D}>\pi^{C}$ in the proof of Proposition 4 shows that $C$ is optimal if $c<\frac{2}{3} \lambda \rho h$ and $\lambda \rho h<\frac{k}{2}$ (i.e., $h<\hat{h}$ ). According to the second line of $\pi^{D}>\pi_{k>c}^{P D}$ in the proof of Proposition 4, $C$ will dominate $P D$ if $c<\frac{k}{2} \leq \lambda \rho h<k$ but $\frac{2}{3}(k-\lambda \rho h)>c \Leftrightarrow k-\frac{3}{2} c>\lambda \rho h$. Hence, $c$ has to be sufficiently small. Since we must have $\frac{k}{2} \leq \lambda \rho h<k$, only those parameter constellations are feasible that satisfy $k-\frac{3}{2} c \geq \frac{k}{2} \Leftrightarrow c \leq \frac{k}{3}$. Therefore, $c<\frac{k}{2} \leq \lambda \rho h<k$ (implying $h<\bar{h}$ ) with $c \leq \frac{k}{3}$ describes a second region where $C$ dominates $P D$. In all remaining regions, $P D$ is the optimal organizational structure.

\section{Proof of Proposition 5:}

Comparing expected profits for $c<k$ and $h<q$ yields $^{8}$

$$
\pi^{C A}>\pi^{C} \Leftrightarrow\left\{\begin{array}{c}
\text { if } \quad c>\frac{k}{2} \\
\text { if } c<\frac{k}{2} \leq \lambda \rho q \text { then } \quad \frac{1}{3} k<c \\
\text { if } c \leq \lambda \rho q<\frac{k}{2} \text { then } \frac{2}{3} \lambda \rho q<c \\
\text { if } \lambda \rho q<c<\frac{k}{2}
\end{array}\right.
$$

\footnotetext{
${ }^{8}$ See the Additional Pages for the Referees.
} 
$\pi^{C A}>\pi_{k>c}^{P D} \Leftrightarrow\left\{\begin{array}{c}\text { if } c<k \leq \lambda \rho h<\lambda \rho q \quad \text { then } \quad 2(q-h) \rho>\frac{2}{3} k-c \\ \text { if } \lambda \rho q \geq c \text { and } c \leq \lambda \rho h<k \text { then } 2(q-h) \rho>\frac{2}{3} \lambda \rho h-c \\ \text { if } \lambda \rho h<c \text { and } \lambda \rho q<k\end{array}\right.$

Let $\frac{k}{2}<c<k$ and $h<q$. Then

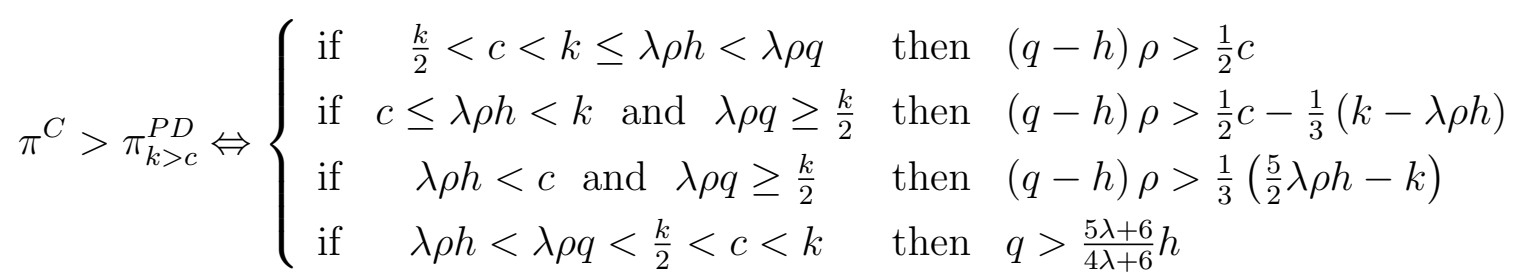

Let $c<\frac{k}{2}<k$ and $h<q$. Then

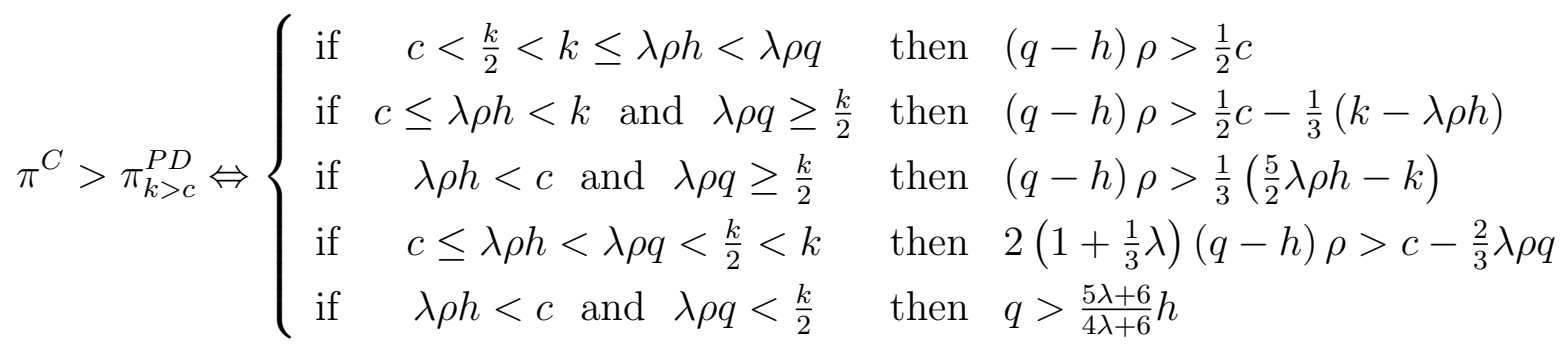

The statement on the optimality of cross-authority delegation follows from $\pi^{C A}>\pi^{C}$ since the conditions $\frac{1}{3} k<c$ and $\frac{2}{3} \lambda \rho q<c$ (with $\lambda \rho q<\frac{k}{2}$ ) are satisfied for $c>\frac{2}{3} k$, and from $\pi^{C A}>\pi_{k>c}^{P D}$ since the conditions $\frac{2}{3} k-$ $c<0$ and $\frac{2}{3} \lambda \rho h-c<0$ (with $\lambda \rho h<k$ ) hold for $c>\frac{2}{3} k$. Optimality of partial delegation for the stated parameter constellations can be seen from the following observations: If $c<\frac{2}{3} k$ and $h \geq \bar{h}$ is close to $q$, then conditions $2(q-h) \rho>\frac{2}{3} k-c$ and $2(q-h) \rho>\frac{2}{3} \lambda \rho h-c$ in lines 1 and 2 of $\pi^{C A}>\pi_{k>c}^{P D}$ and condition $\lambda \rho q<k$ in the third line of $\pi^{C A}>\pi_{k>c}^{P D}$ will be violated; similarly, $\pi^{C}>\pi_{k>c}^{P D}$ is violated under the conditions stated in Proposition 5 . Concerning centralization, the profit comparisons above immediately show that $\pi^{C A}<\pi^{C}$ and $\pi^{C}>\pi_{k>c}^{P D}$ for $h<\bar{h}$ and sufficiently small values of $c$.

\section{Construction of Figure 2:}

Note that $\pi^{C A}>\pi_{k>c}^{P D}$ in the proof of Proposition 5 is always satisfied for $c>\frac{2}{3} k$ since in the first line $\frac{1}{3} k-\frac{1}{2} c<0$ and in the second line (where 
$\lambda \rho h<k$ must hold) $\frac{2}{3} \lambda \rho h-c<0$ under $c>\frac{2}{3} k$. As $\pi^{C A}>\pi^{C}$ for $c>\frac{k}{2}$, the organizational structure $C A$ will be optimal if $c>\frac{2}{3} k$. The third line of the comparison $\pi^{C A}>\pi_{k>c}^{P D}$ in the proof of Proposition 5 shows that $C A$ dominates $P D$ for all points in Figure 2 that lie above the horizontal line $c=\lambda \rho h$ and, at the same time, left to the vertical line through $\bar{q}$ being defined by $\lambda \bar{q} \rho=k$. According to $\pi^{C A}>\pi^{C}$ in the proof of Proposition $5, c>\frac{1}{3} k$ is sufficient for $C A$ to dominate $C$ (note that in the third line of $\pi^{C A}>\pi^{C}$ the inequality $\frac{2}{3} \lambda \rho q<c$ is satisfied under $c>\frac{1}{3} k$ since $\left.\lambda \rho q<\frac{k}{2}\right)$. Thus, $C A$ is the optimal organizational structure if $c>\frac{1}{3} k$ and $q<\bar{q}$. According to the third line and the fourth line of $\pi^{C A}>\pi^{C}$ in the proof of Proposition 5, $C A$ will dominate $C$ if $c>\frac{2}{3} \lambda \rho q$ and $q<\hat{q}$ with $\hat{q}$ being defined by $\lambda \hat{q} \rho=\frac{k}{2}$. For the same parameter constellations, $C A$ also dominates $P D$ as is shown by the second line and the third line of $\pi^{C A}>\pi_{k>c}^{P D}$ in the proof of Proposition 5 .

For the remainder of the parameter space, either $C$ or $P D$ is optimal. If $\frac{k}{2}<c<k$, according to our previous findings for Figure 2, only parameter constellations with $c \in\left(\frac{k}{2}, \frac{2}{3} k\right)$ and $q>\bar{q}$ are relevant. According to the third line of $\pi^{C}>\pi_{k>c}^{P D}$ in the proof of Proposition 5, organizational structure $C$ dominates structure $P D$ because, for $q>\bar{q}$,

$$
(q-h) \rho>\frac{1}{3}\left(\frac{5}{2} \lambda \rho h-k\right) \Leftrightarrow q>h+\frac{5}{6} \lambda h-\frac{1}{3 \rho} k
$$

is satisfied since

$$
h+\frac{5}{6} \lambda h-\frac{1}{3 \rho} k<\bar{q}=\frac{k}{\lambda \rho} \Leftrightarrow \lambda \rho h<2 \frac{\lambda+3}{5 \lambda+6} k
$$

is true because of $2 \frac{\lambda+3}{(5 \lambda+6)} \in(0.72727,1)$.

Now, we consider the parameter space satisfying $c<\frac{k}{2}<k$. By using the same argumentation as before, we immediately have that $C$ is optimal for $c>\lambda \rho h$ and $q>\bar{q}$. Moreover, since even

$$
h+\frac{5}{6} \lambda h-\frac{1}{3 \rho} k<\hat{q}=\frac{k}{2 \lambda \rho} \Leftrightarrow \lambda \rho h<\frac{2 \lambda+3}{5 \lambda+6} k
$$

is satisfied as $\frac{2 \lambda+3}{5 \lambda+6} \in(0.45455,0.5)$, organizational structure $C$ is also optimal for $c>\lambda \rho h$ and $q \in(\hat{q}, \bar{q})$. The second line of $\pi^{C}>\pi_{k>c}^{P D}$ deals with the case 
of $c \leq \lambda \rho h$ and $\lambda \rho q \geq \frac{k}{2} \Leftrightarrow q \geq \hat{q}$. Recall that $c<\frac{k}{3}$. Since

$$
(q-h) \rho>\frac{1}{2} c-\frac{1}{3}(k-\lambda \rho h)
$$

is even true for $c=\frac{k}{3}$, i.e.,

$$
\begin{aligned}
(q-h) \rho & >\frac{1}{2} \cdot \frac{k}{3}-\frac{1}{3}(k-\lambda \rho h)=\frac{1}{3} \lambda \rho h-\frac{1}{6} k \Leftrightarrow \\
q & >\frac{1}{3} \lambda h-\frac{1}{6 \rho} k+h
\end{aligned}
$$

holds because

$$
\frac{1}{3} \lambda h-\frac{1}{6 \rho} k+h<\hat{q}=\frac{k}{2 \lambda \rho} \Leftrightarrow \lambda \rho h<\frac{k}{2}
$$

is true, organizational structure $C$ is optimal if $c \leq \lambda \rho h$ and $q \geq \hat{q}$. Finally, the last two lines of $\pi^{C}>\pi_{k>c}^{P D}$ show that $C$ is optimal for $c<\frac{2}{3} \lambda \rho q$ and $q<\hat{q}$.

\section{Construction of Figure 3:}

From Proposition 3 we know that $C A$ is optimal for $c>k$, and the proof of Proposition 5 shows that $C A$ is optimal for $c>\frac{2}{3} k$. The first line and the second line of $\pi^{C A}>\pi^{C}$ in the proof of Proposition 5 point out that $C A$ dominates $C$ for $c \in\left(\frac{k}{3}, \frac{2}{3} k\right)$. From the first line of $\pi^{C A}>\pi_{k>c}^{P D}$ in the proof of Proposition 5 we obtain that $C A$ dominates $P D$ iff

$$
2(q-h) \rho>\frac{2}{3} k-c \Leftrightarrow c>\frac{2}{3} k+2 \rho h-2 \rho q .
$$

Note that the right-hand side of the last inequality describes a straight line with slope $-2 \rho$ that intersects the vertical axis in the point $\left(h, \frac{2}{3} k\right)$ and the horizontal axis in the point $\left(h+\frac{k}{3 \rho}, 0\right)$. Thus, the region above the line $\frac{2}{3} k+2 \rho h-2 \rho q$ corresponds to parameter constellations for which $C A$ dominates $P D$, whereas points below the line describe situations in which $P D$ dominates $C A$. Altogether, $C A$ is the optimal organizational structure if $c>\frac{2}{3} k+2 \rho h-2 \rho q$ and $q \in\left[h, h+\frac{k}{6 \rho}\right]$, or if $c>\frac{k}{3}$ and $q>h+\frac{k}{6 \rho}$. If $\frac{2}{3} k+2 \rho h-2 \rho q<c<\frac{k}{3}$ and $q>h+\frac{k}{6 \rho}$, then owner $O$ prefers $C A$ to $P D$ but also $C$ to $C A$ so that the organizational structure $C$ is optimal. Finally, the 
first line of $\pi^{C}>\pi_{k>c}^{P D}$ in the proof of Proposition 5 shows that $C$ dominates $P D$ iff

$$
(q-h) \rho>\frac{1}{2} c \Leftrightarrow c<2 \rho q-2 \rho h,
$$

where the right-hand side of the last inequality describes a straight line with slope $2 \rho$ that intersects both $c=\frac{2}{3} k+2 \rho h-2 \rho q$ and $c=\frac{k}{3}$ in the point $\left(h+\frac{k}{6 \rho}, \frac{k}{3}\right)$. Therefore, $C$ is optimal if $c<2 \rho q-2 \rho h$ and $q \in\left[h, h+\frac{k}{6 \rho}\right]$, or if $c<\frac{2}{3} k+2 \rho h-2 \rho q$ and $q \in\left(h+\frac{k}{6 \rho}, h+\frac{k}{3 \rho}\right]$. For the remaining parameter space with $2 \rho q-2 \rho h<c<\frac{2}{3} k+2 \rho h-2 \rho q$ and $q \in\left[h, h+\frac{k}{6 \rho}\right]$, organizational structure $P D$ is optimal.

\section{References}

Aghion, P., Dewatripont, M., and P. Rey (2002): On Partial Contracting. European Economic Review 46, 745-753.

Aghion, P. and J. Tirole (1997): Formal and Real Authority in Organizations. Journal of Political Economy 105, 1-29.

Alonso, R., Dessein W., and N. Matouschek (2008): When Does Coordination Require Centralization?. American Economic Review 98, 145-179.

Chandler, A.D. (1977): The Visible Hand: The Managerial Revolution in American Business. Harvard University Press: Cambridge, MA.

Che, Y.-K. and S.-W. Yoo (2001): Optimal Incentives for Teams. American Economic Review 91, 525-541.

Choe, C. and S. Ishiguro (2012): On the Optimality of Multi-tier Hierarchies: Coordination versus Motivation. Journal of Law, Economics, and Organization 28, 486-517.

Demougin, D. and C. Fluet (1998): Mechanism Sufficient Statistic in the Risk-Neutral Agency Problem. Journal of Institutional and Theoretical Economics 154, 622-639. 
Dessein, W. (2002): Authority and Communication in Organizations. Review of Economic Studies 69, 811-838.

Dessein, W. (forthcoming): Incomplete Contracts and Firm Boundaries: New Directions. Journal of Law, Economics, and Organization.

Hermalin, B.E. (2005): Lecture Notes for Economics.

Innes, R.D. (1990): Limited Liability and Incentive Contracting with ExAnte Action Choices. Journal of Economic Theory 52, 45-67.

Kräkel, M. and A. Schöttner (2012): Internal Labor Markets and Worker Rents. Journal of Economic Behavior and Organization 84, 491-509.

Laffont, J.-J. and D. Martimort (2002): The Theory of Incentives. Princeton University Press: Princeton and Oxford.

Milgrom, P.R. and J. Roberts (199s): Economics, Organization and Management. Prentice-Hall: Englewood Cliffs.

Milgrom, P.R. and J. Roberts (1995a): Continuous Adjustment and Fundamental Change in Business Strategy and Organization, in: Siebert, H. (ed.): Trends in Business Organization: Do Participation and Cooperation Increase Competitiveness?. Mohr Siebeck: Tübingen, 231-258.

Milgrom, P.R. and J. Roberts (1995b): Complementarities and Fit: Strategy, Structure, and Organizational Change in Manufacturing. Journal of Accounting and Economics 19, 179-208.

Ohlendorf, S. and P.W. Schmitz (2012): Repeated Moral Hazard and Contracts with Memory: The Case of Risk-Neutrality. International Economic Review 53, 433-452.

Rantakari, H. (2008): Governing Adaptation. Review of Economic Studies 75, 1257-1285. 
Sappington, D. (1983): Limited Liability Contracts between Principal and Agent. Journal of Economic Theory 29, 1-21.

Schmitz, P.W. (2005a): Allocating Control in Agency Problems with Limited Liability and Sequential Hidden Actions. RAND Journal of Economics 36, 318-336.

Schmitz, P.W. (2005b): Should Contractual Clauses that Forbid Renegotiation Always be Enforced?. Journal of Law, Economics, and Organization 21, 315-329.

Schmitz, P.W. (2013): Job Design with Conflicting Tasks Reconsidered. European Economic Review 57, 108-117.

Thiele, V. (2013): Subjective Performance Evaluations, Collusion, and Organizational Design. Journal of Law, Economics, and Organization 29, $35-59$.

Williamson, O.E. (1981): The Modern Corporation: Origins, Evolution, Attributes. Journal of Economic Literature 19, 1537-1568. 


\section{Additional Pages for the Referees}

Additional material for the proof of Proposition 4:

Let $h>q$ and $c \in(k / 2, k)$. Then the comparison of $\pi^{D}$ and $\pi^{C}$ yields: If $\frac{k}{2 \rho}<\frac{c}{\rho} \leq \lambda h$, then $\pi^{D}>\pi^{C} \Leftrightarrow 4 h \rho-\frac{4}{3} k>4(h \rho-c) \Leftrightarrow \frac{1}{3} k<c$, which is true since $c>k / 2$. If $\frac{k}{2 \rho} \leq \lambda h<\frac{c}{\rho}$, then $\pi^{D}>\pi^{C} \Leftrightarrow(4+4 \lambda) h \rho-4 c-\frac{4}{3} k>$ $4(h \rho-c) \Leftrightarrow \lambda \rho h-\frac{1}{3} k>0$, which is true since $\frac{k}{2 \rho} \leq \lambda h$. If $\lambda h<\frac{k}{2 \rho}<\frac{c}{\rho}$, then $\pi^{D}>\pi^{C} \Leftrightarrow(4+4 \lambda) h \rho-4 c-\frac{4}{3} k>\left(4+\frac{8}{3} \lambda\right) h \rho-4 c-\frac{4}{3} k \Leftrightarrow 4 \lambda>\frac{8}{3} \lambda$ is true. The comparison of $\pi_{k>c}^{P D}$ and $\pi^{C}$ leads to the following results: If $\frac{k}{2 \rho}<\frac{c}{\rho}<\frac{k}{\rho} \leq \lambda h$, then $\pi_{k>c}^{P D}>\pi^{C} \Leftrightarrow 4 h \rho-2 c>4(h \rho-c)$ is true. If $\frac{k}{2 \rho}<\frac{c}{\rho} \leq \lambda h<\frac{k}{\rho}$, then $\pi_{k>c}^{P D}>\pi^{C} \Leftrightarrow\left(4+\frac{4}{3} \lambda\right) h \rho-2 c-\frac{4}{3} k>4(h \rho-c) \Leftrightarrow$ $\left(\lambda \rho h-\frac{1}{2} k\right)+\left(\frac{3}{2} c-\frac{k}{2}\right)>0$, which is true since $\frac{k}{2 \rho}<\lambda h$ and $c>k / 2$. If $\frac{k}{2 \rho} \leq \lambda h<\frac{c}{\rho}<\frac{k}{\rho}$, then $\pi_{k>c}^{P D}>\pi^{C} \Leftrightarrow\left(4+\frac{10}{3} \lambda\right) h \rho-4 c-\frac{4}{3} k>4(h \rho-c) \Leftrightarrow$ $\lambda \rho h-\frac{2}{5} k>0$ is true since $\frac{k}{2 \rho} \leq \lambda h$. If $\lambda h<\frac{k}{2 \rho}<\frac{c}{\rho}<\frac{k}{\rho}$, then $\pi_{k>c}^{P D}>\pi^{C} \Leftrightarrow$ $\left(4+\frac{10}{3} \lambda\right) h \rho-4 c-\frac{4}{3} k>\left(4+\frac{8}{3} \lambda\right) h \rho-4 c-\frac{4}{3} k \Leftrightarrow \frac{10}{3} \lambda>\frac{8}{3} \lambda$ is true.

Now, let $h>q$ but $c<k / 2$. Comparing organizational structures $D$ and $C$ gives the following results: If $\frac{c}{\rho}<\frac{k}{2 \rho} \leq \lambda h$, then $\pi^{D}>\pi^{C}$ is true iff $4 h \rho-\frac{4}{3} k>4(h \rho-c) \Leftrightarrow \frac{1}{3} k<c$. If $\frac{c}{\rho} \leq \lambda h<\frac{k}{2 \rho}$, then $\pi^{D}>\pi^{C}$ is true iff $4 h \rho-\frac{4}{3} k>\left(4+\frac{8}{3} \lambda\right) h \rho-4 c-\frac{4}{3} k \Leftrightarrow c>\frac{2}{3} \lambda \rho h$. If $\lambda h<\frac{c}{\rho}<\frac{k}{2 \rho}$, then $\pi^{D}>\pi^{C} \Leftrightarrow(4+4 \lambda) h \rho-4 c-\frac{4}{3} k>\left(4+\frac{8}{3} \lambda\right) h \rho-4 c-\frac{4}{3} k \Leftrightarrow 4 \lambda>\frac{8}{3} \lambda$ is true. The comparison of $P D$ and $C$ yields: If $\frac{c}{\rho}<\frac{k}{2 \rho}<\frac{k}{\rho} \leq \lambda h$, then $\pi_{k>c}^{P D}>$ $\pi^{C} \Leftrightarrow 4 h \rho-2 c>4(h \rho-c) \Leftrightarrow 2 c<4 c$ is true. If $\frac{c}{\rho}<\frac{k}{2 \rho} \leq \lambda h<\frac{k}{\rho}$, then $\pi_{k>c}^{P D}>\pi^{C}$ is true iff $\left(4+\frac{4}{3} \lambda\right) h \rho-2 c-\frac{4}{3} k>4(h \rho-c) \Leftrightarrow c>\frac{2}{3}(k-\lambda \rho h)$. If $\frac{c}{\rho} \leq \lambda h<\frac{k}{2 \rho}<\frac{k}{\rho}$, then $\pi_{k>c}^{P D}>\pi^{C}$ is true iff $\left(4+\frac{4}{3} \lambda\right) h \rho-2 c-\frac{4}{3} k>$ $\left(4+\frac{8}{3} \lambda\right) h \rho-4 c-\frac{4}{3} k \Leftrightarrow c>\frac{2}{3} \lambda \rho h$. If $\lambda h<\frac{c}{\rho}<\frac{k}{2 \rho}<\frac{k}{\rho}$, then $\pi_{k>c}^{P D}>\pi^{C} \Leftrightarrow$ $\left(4+\frac{10}{3} \lambda\right) h \rho-4 c-\frac{4}{3} k>\left(4+\frac{8}{3} \lambda\right) h \rho-4 c-\frac{4}{3} k \Leftrightarrow \frac{10}{3} \lambda>\frac{8}{3} \lambda$ is true.

Finally, we have to compare $D$ and $P D$ under $h>q$ without restricting $c$ : If $\frac{c}{\rho}<\frac{k}{\rho} \leq \lambda h$, then $\pi^{D}>\pi_{k>c}^{P D}$ is true iff $4 h \rho-\frac{4}{3} k>4 h \rho-2 c \Leftrightarrow \frac{2}{3} k<c$. If $\frac{c}{\rho} \leq \lambda h<\frac{k}{\rho}$, then $\pi^{D}>\pi_{k>c}^{P D}$ is true iff $4 h \rho-\frac{4}{3} k>\left(4+\frac{4}{3} \lambda\right) h \rho-2 c-\frac{4}{3} k \Leftrightarrow$ $c>\frac{2}{3} \lambda \rho h$. If $\lambda h<\frac{c}{\rho}<\frac{k}{\rho}$, then $\pi^{D}>\pi_{k>c}^{P D} \Leftrightarrow(4+4 \lambda) h \rho-4 c-\frac{4}{3} k>$ $\left(4+\frac{10}{3} \lambda\right) h \rho-4 c-\frac{4}{3} k \Leftrightarrow 4 \lambda>\frac{10}{3} \lambda$ is true. 
Additional material for the proof of Proposition 5:

Let $c>k / 2$. If $\frac{k}{2 \rho}<\frac{c}{\rho} \leq \lambda q$, then $\pi^{C A}>\pi^{C} \Leftrightarrow 4 q \rho-\frac{4}{3} k>4(q \rho-c) \Leftrightarrow$ $\frac{1}{3} k<c$, which is true since $c>k / 2$. If $\frac{k}{2 \rho} \leq \lambda q<\frac{c}{\rho}$, then $\pi^{C A}>\pi^{C} \Leftrightarrow$ $(4+4 \lambda) q \rho-4 c-\frac{4}{3} k>4(q \rho-c) \Leftrightarrow \lambda \rho q>\frac{1}{3} k$ is true since $\frac{k}{2 \rho} \leq \lambda q$. If $\lambda q<\frac{k}{2 \rho}<\frac{c}{\rho}$, then $\pi^{C A}>\pi^{C} \Leftrightarrow(4+4 \lambda) q \rho-4 c-\frac{4}{3} k>\left(4+\frac{8}{3} \lambda\right) q \rho-4 c-$ $\frac{4}{3} k \Leftrightarrow 4 \lambda>\frac{8}{3} \lambda$ is true. Altogether, if $c>k / 2$ then $C A$ will dominate $C$.

Now, let $c<k / 2$. If $\frac{c}{\rho}<\frac{k}{2 \rho} \leq \lambda q$, then $\pi^{C A}>\pi^{C} \Leftrightarrow 4 q \rho-\frac{4}{3} k>$ $4(q \rho-c) \Leftrightarrow \frac{1}{3} k<c$. If $\frac{c}{\rho} \leq \lambda q<\frac{k}{2 \rho}$, then $\pi^{C A}>\pi^{C} \Leftrightarrow 4 q \rho-\frac{4}{3} k>$ $\left(4+\frac{8}{3} \lambda\right) q \rho-4 c-\frac{4}{3} k \Leftrightarrow c>\frac{2}{3} \lambda \rho q$. If $\lambda q<\frac{c}{\rho}<\frac{k}{2 \rho}$, then $\pi^{C A}>\pi^{C} \Leftrightarrow$ $(4+4 \lambda) q \rho-4 c-\frac{4}{3} k>\left(4+\frac{8}{3} \lambda\right) q \rho-4 c-\frac{4}{3} k \Leftrightarrow 4 \lambda>\frac{8}{3} \lambda$, which is true.

Next, we can compare $C A$ and $P D$.

If $\frac{c}{\rho}<\frac{k}{\rho} \leq \lambda h<\lambda q$, then $\pi^{C A}>\pi_{k>c}^{P D} \Leftrightarrow 4 q \rho-\frac{4}{3} k>4 h \rho-2 c \Leftrightarrow$ $(q-h) \rho>\frac{1}{3} k-\frac{1}{2} c$.

If $\frac{c}{\rho}<\lambda h<\frac{k}{\rho} \leq \lambda q$ or if $\frac{c}{\rho} \leq \lambda h<\lambda q<\frac{k}{\rho}$, then $\pi^{C A}>\pi_{k>c}^{P D} \Leftrightarrow 4 q \rho-\frac{4}{3} k>$ $\left(4+\frac{4}{3} \lambda\right) h \rho-2 c-\frac{4}{3} k \Leftrightarrow(q-h) \rho>\frac{1}{3} \lambda \rho h-\frac{1}{2} c$. If $\lambda h<\frac{c}{\rho}<\frac{k}{\rho} \leq \lambda q$ or if $\lambda h<\frac{c}{\rho} \leq \lambda q<\frac{k}{\rho}$, then $\pi^{C A}>\pi_{k>c}^{P D} \Leftrightarrow 4 q \rho-\frac{4}{3} k>\left(4+\frac{10}{3} \lambda\right) h \rho-4 c-\frac{4}{3} k \Leftrightarrow$ $(q-h) \rho>\frac{5}{6} \lambda \rho h-c$, which is true since $\lambda h<\frac{c}{\rho}$. If $\lambda h<\lambda q<\frac{c}{\rho}<\frac{k}{\rho}$, then $\pi^{C A}>\pi_{k>c}^{P D} \Leftrightarrow(4+4 \lambda) q \rho-4 c-\frac{4}{3} k>\left(4+\frac{10}{3} \lambda\right) h \rho-4 c-\frac{4}{3} k \Leftrightarrow(4+4 \lambda) q \rho>$ $\left(4+\frac{10}{3} \lambda\right) h \rho$ is true. The findings can be condensed to the results presented in the proof of Proposition 5.

Finally, we have to compare $C$ and $P D$. Let $c>k / 2$. If $\frac{k}{2 \rho}<\frac{c}{\rho}<\frac{k}{\rho} \leq$ $\lambda h<\lambda q$, then $\pi^{C}>\pi_{k>c}^{P D} \Leftrightarrow 4(q \rho-c)>4 h \rho-2 c \Leftrightarrow(q-h) \rho>\frac{1}{2} c$. If $\frac{k}{2 \rho}<\frac{c}{\rho}<\lambda h<\frac{k}{\rho} \leq \lambda q$ or if $\frac{k}{2 \rho}<\frac{c}{\rho} \leq \lambda h<\lambda q<\frac{k}{\rho}$, then $\pi^{C}>\pi_{k>c}^{P D} \Leftrightarrow$ $4(q \rho-c)>\left(4+\frac{4}{3} \lambda\right) h \rho-2 c-\frac{4}{3} k \Leftrightarrow(q-h) \rho>\frac{1}{2} c-\frac{1}{3}(k-\lambda \rho h)$. If $\frac{k}{2 \rho}<$ $\lambda h<\frac{c}{\rho}<\frac{k}{\rho} \leq \lambda q$ or if $\lambda h<\frac{k}{2 \rho}<\frac{c}{\rho}<\frac{k}{\rho} \leq \lambda q$ or if $\frac{k}{2 \rho}<\lambda h<\frac{c}{\rho} \leq \lambda q<\frac{k}{\rho}$ or if $\lambda h<\frac{k}{2 \rho}<\frac{c}{\rho} \leq \lambda q<\frac{k}{\rho}$ or if $\frac{k}{2 \rho} \leq \lambda h<\lambda q<\frac{c}{\rho}<\frac{k}{\rho}$ or if $\lambda h<\frac{k}{2 \rho} \leq$ $\lambda q<\frac{c}{\rho}<\frac{k}{\rho}$, then $\pi^{C}>\pi_{k>c}^{P D} \Leftrightarrow 4(q \rho-c)>\left(4+\frac{10}{3} \lambda\right) h \rho-4 c-\frac{4}{3} k \Leftrightarrow$ $(q-h) \rho>\frac{1}{3}\left(\frac{5}{2} \lambda \rho h-k\right)$. If $\lambda h<\lambda q<\frac{k}{2 \rho}<\frac{c}{\rho}<\frac{k}{\rho}$, then $\pi^{C}>\pi_{k>c}^{P D} \Leftrightarrow$ $\left(4+\frac{8}{3} \lambda\right) q \rho-4 c-\frac{4}{3} k>\left(4+\frac{10}{3} \lambda\right) h \rho-4 c-\frac{4}{3} k \Leftrightarrow\left(1+\frac{2}{3} \lambda\right) q \rho>\left(1+\frac{5}{6} \lambda\right) h \rho$. Again, the findings can be condensed to the results presented in the proof of Proposition 5. 
Now, let $c<k / 2$.

If $\frac{c}{\rho}<\frac{k}{2 \rho}<\frac{k}{\rho} \leq \lambda h<\lambda q$, then $\pi^{C}>\pi_{k>c}^{P D} \Leftrightarrow 4(q \rho-c)>4 h \rho-2 c \Leftrightarrow$ $(q-h) \rho>\frac{1}{2} c$. If $\frac{c}{\rho}<\frac{k}{2 \rho}<\lambda h<\frac{k}{\rho} \leq \lambda q$ or if $\frac{c}{\rho}<\lambda h<\frac{k}{2 \rho}<\frac{k}{\rho} \leq \lambda q$ or if $\frac{c}{\rho}<\frac{k}{2 \rho} \leq \lambda h<\lambda q<\frac{k}{\rho}$ or if $\frac{c}{\rho}<\lambda h<\frac{k}{2 \rho} \leq \lambda q<\frac{k}{\rho}$, then $\pi^{C}>$ $\pi_{k>c}^{P D} \Leftrightarrow 4(q \rho-c)>\left(4+\frac{4}{3} \lambda\right) h \rho-2 c-\frac{4}{3} k \Leftrightarrow(q-h) \rho>\frac{1}{2} c-\frac{1}{3}(k-\lambda \rho h)$. If $\lambda h<\frac{c}{\rho}<\frac{k}{2 \rho}<\frac{k}{\rho} \leq \lambda q$ or if $\lambda h<\frac{c}{\rho}<\frac{k}{2 \rho} \leq \lambda q<\frac{k}{\rho}$, then $\pi^{C}>$ $\pi_{k>c}^{P D} \Leftrightarrow 4(q \rho-c)>\left(4+\frac{10}{3} \lambda\right) h \rho-4 c-\frac{4}{3} k \Leftrightarrow(q-h) \rho>\frac{1}{3}\left(\frac{5}{2} \lambda \rho h-k\right)$. If $\frac{c}{\rho} \leq \lambda h<\lambda q<\frac{k}{2 \rho}<\frac{k}{\rho}$, then $\pi^{C}>\pi_{k>c}^{P D} \Leftrightarrow\left(4+\frac{8}{3} \lambda\right) q \rho-4 c-\frac{4}{3} k>$ $\left(4+\frac{4}{3} \lambda\right) h \rho-2 c-\frac{4}{3} k \Leftrightarrow\left(1+\frac{1}{3} \lambda\right)(q-h) \rho>\frac{1}{2} c-\frac{1}{3} \lambda \rho q$. If $\lambda h<\frac{c}{\rho} \leq \lambda q<$ $\frac{k}{2 \rho}<\frac{k}{\rho}$ or if $\lambda h<\lambda q<\frac{c}{\rho}<\frac{k}{2 \rho}<\frac{k}{\rho}$, then $\pi^{C}>\pi_{k>c}^{P D} \Leftrightarrow\left(4+\frac{8}{3} \lambda\right) q \rho-4 c-\frac{4}{3} k>$ $\left(4+\frac{10}{3} \lambda\right) h \rho-4 c-\frac{4}{3} k \Leftrightarrow q>\frac{5 \lambda+6}{4 \lambda+6} h$. Condensing the various cases yields the results in the proof of Proposition 5. 